\title{
Imitation-driven Cultural Collapse
}

\section{Salva Duran-Nebredaa, ${ }^{a, *}$ and Sergi Valverde ${ }^{a, *}$}

${ }_{3}$ aEvolution of Technology Lab, Institute of Evolutionary Biology (UPF-CSIC), Passeig de la Barceloneta, E-08003,

4 Barcelona.

${ }_{5}$ *salva.duran@ibe.upf-csic.es; sergi.valverde@ibe.upf-csic.es

\section{ABSTRACT}

A unique feature of human society is its ever-increasing diversity and complexity. Although individuals can generate meaningful variation, they can never create the whole cultural and technological knowledge sustaining a society on their own. Instead, social transmission of innovations accounts for most of the accumulated human knowledge. The natural cycle of cumulative culture entails fluctuations in the diversity of artifacts and behaviours, with sudden declines unfolding in particular domains. Cultural collapses have been attributed to exogenous causes. Here, we define a theoretical framework to explain endogenous cultural collapses, driven by an exploration-reinforcement imbalance. This is demonstrated by the historical market crash of the Atari Video Console System in 1983, the cryptocurrency crash of 2018 and the production of memes in online communities. We identify universal features of endogenous cultural collapses operating at different scales, including changes in the distribution of component usage, higher compressibility of the cultural history, marked decreases in complexity and the boom-and-bust dynamics of diversity. The loss of information is robust and independent of specific system details. Our framework suggests how future endogenous collapses can be mitigated by stronger self-policing, reduced costs to innovation and increased transparency of cost-benefit trade-offs.

\section{Introduction} capacity for high-fidelity copying ${ }^{1,2}$. Many studies have studied the relationship between individual and social learning and 
imitation-driven collapse is not new, current approaches cannot easily predict collapses. Obstacles include an emphasis on unobserved exogenous factors (e.g., environmental fluctuations), lack of empirical studies, and abstract models. Here, we aim to fill this gap by defining a robust signature of imitation-driven cultural collapses, i.e., to predict when accumulation of redundant information impedes further cultural diversification.

Common features of cultural collapses are still poorly understood. Few studies have examined the loss of cultural information with empirical data ${ }^{9}$. We reconstruct (and analyze) cultural diversity dynamics in different empirical systems, including: (1) Atari 2600 and (2) Commodore Vic-20 video games, (3) cryptocurrency white-papers, (4) scientific publications, and social memes from two (5) Reddit communities (see Methods). Each system is an evolving population of cultural artifacts, where the current generation of novelties is influenced by prior artifacts through a shared pool of components. None of these systems have been simultaneously studied at the artifact-genotype scale (e.g., binary or lexicographic sequences), structural scale (network of component associations), and global scale (full corpus). We rely on standard methods of linguistic analysis ${ }^{11-13}$, but reliable extraction of genotypes involves new reverse-engineering techniques exploiting phenotypic information (see Methods).

A mechanistic model of evolutionary dynamics formalizes our hypothesis. Polya's urn can simulate recombination and tinkering, which are often proposed as drivers of technological innovation ${ }^{14,15}$. A generalization of the Polya's urn has been shown to predict the statistical laws at which novelties happen and the probability distribution of components ${ }^{16,17}$. We extend this model to show how recombination of existing components does not always translate to innovations when the space of designs is crowded with highly-similar products. Saturation imposed by redundant components could be followed by a cultural collapse, i.e. a sharp reduction in diversity. This suggests how cultural collapses can take place even in the absence of external forces, i.e., they are an emergent property of innovation-reinforcement imbalances.

Culture is a complex dynamic system involving multiple causal levels. However, a large family of models represent culture with a small number of traits or components, limiting their applicability ${ }^{18}$. Large-scale changes in cultural and technological systems actually depend on many component frequencies and their interactions. We observe the pattern of cultural collapse at multiple scales and using different metrics: (a) component usage frequency ${ }^{16}$, (b) corpus compressibility ${ }^{19}$ and (c) structural artifact complexity ${ }^{13}$. We assessed the significance of our observations using negative controls (i.e., a comparable empirical system not showing endogenous cultural collapse) and the mechanistic model. The reported global pattern in the loss of innovation is robust and independent of specific system details. We conclude with an analysis of how imitation-driven cultural collapses can be avoided.

\section{Results and discussion}

\section{Boom-and-bust dynamics in artifact productivity}

Recently, it was reported that the rate of cultural evolution declines over long time intervals, due to some intrinsic constraints ${ }^{20}$. An empirical analysis of cultural systems reveals that the pace of innovation can change very rapidly as well. A consistent trend is the diversity $(N(t))$ of ideas and artifacts has been accelerating in time ${ }^{21}$. These patterns are not associated with 
random fluctuations, but with the mechanisms of cumulative cultural evolution (see below). Studies of cultural evolution have naturally focused on the expansion of diversity, but much less attention has been devoted to the decline of cultural diversity and determining eventual cultural collapses.

Figure 1A shows the number $N(t)$ of almost every released video game for the Atari Video Computer System (later renamed to Atari 2600), from 1977 to 1992. This data set comprises $N=\sum N(t)=551$ different Atari 2600 cartridges containing the assembler or machine game codes. The boom and bust dynamics in video game diversity maps a large-scale recession (also called the "video game crash of 1983") that took place from 1983 to $1985^{22}$. This collapse has been related to external factors: (1) saturation in the number of game consoles and available games, (2) competition between game consoles and personal computers $^{22}$, (3) poor quality of third-party games that devalued the market ${ }^{23}$. Many games released by third-party developers imitated (and in some cases even cloned, see Figure 1a) successful titles, e.g, Space Invaders (Atari, 1980).

A very similar phenomenon has been recently observed in the cryptocurrency market. In Figure 1b we show the number $N(t)$ of cryptocurrencies released each year from 2009 to 2020. Presumably, many more cryptocurrency projects have existed, however, documentation in the form of a parseable "white paper" could be only found for the $N=\sum N(t)=1383$ included in this study (see SI). In 2017 there was a huge explosion of cultural diversity, with cryptocurrencies being created for many different purposes: agriculture, finance, real estate development, gambling, etc. This sudden burst has been attributed to neutral diffusion process in a technological landscape ${ }^{24}$ as well as to the launch of Ethereum two years prior, which lowered the entry barrier to creating new currencies with the use of smart contracts ${ }^{25}$. However, the newfound easiness to create a currency might have attracted low-effort creators that did not have the technical know-how to deploy a novel product. The following year marks the burst of the crypto market bubble, with Bitcoin losing $80 \%$ of its value (from $\sim 20.000 \$$ to $\sim 4.000 \$$ ) over the course of $2018^{26}$. Despite the economic downturn, 2018 was the year of maximum cultural productivity, followed by a significant decrease in the rate at which new cryptocurrencies were released.

Figure 1c shows a similar boom-and-bust behaviour for the production of posts in an online community geared towards meme and pun production (subreddit r/PunPatrol). Reddit users in r/PunPatrol post very short text-based jokes and memes ${ }^{27}$, using community specific language and social norms that feature strong social transmission. The dynamics of reinforcement are majorly driven by the user voting as well as the site algorithm for content visualization, which has been linked to the production of less innovative and controversial posts ${ }^{28}$. In this data set, cultural products are $N=\sum N(t)=10761$ collated posts and comments, accumulated over a period of 330 days starting in October 10th, 2018. After a maximum cultural productivity centered around day 150, the number of posts in the subreddit dropped sharply, with a reduced lingering activity.

\section{Polya urn model of innovation}

We model the process of cumulative cultural evolution as a Polya urn model, in which new products are assembled by a population of agents combining components from an underlying distribution (see below). Consider a population of agents exploring a technological space, and constructing new cultural products by combining lower-level components. Following ${ }^{16}$, the process of innovation corresponds to a sampling process of a common set of components, which represent ideas or technologies 
that support a new cultural product (see Figure 2). The composition of this common reservoir of ideas or technologies changes with the creation of new cultural products. Using a component in a cultural product signals its utility to the other creators, reinforcing the likelihood of it being used in the future (a positive feedback mechanism). Additionally, if the chosen component has never been used in the past, new elements are introduced in the distribution, simulating the discovery of new technologies or ideas adjacent to the former. These two processes constitute the backbone of technological creation by composition, and are governed by the amount of reinforcement ( $p$, i.e. how many copies of the same element are added upon using a component) and the amount of exploration $(v+1$, i.e how many new components are introduced after observing an element for the first time) (see SI for a detailed description of the algorithm).

\section{Changes in the Zipf's distribution of component usage}

As reported previously, Polya urn component distribution depends on the rates of reinforcement $(p)$ versus exploration $(v)^{16}$, following a power law with exponent $p / v$. Since cultural products are assembled using a sampling process of the urn, these also reflect the internal component distributions of the urn at the time of the sampling. Consequently, word distributions in cultural products can be used to estimate $p / v$. It has been proposed that natural texts display two different exponents ${ }^{29,30}$, corresponding to a kernel lexicon present in all communications and a domain specific lexicon for the higher ranks that reflects the topic and nature of the communication ${ }^{11}$. However, it has been previously shown that Zipf's law characterization is highly dependent on corpus-size ${ }^{31,32}$. In order to overcome this limitation, we use a control data set for each separate domain, written in the same language as its boom-and-bust counterpart and, with similar format and purpose.

Using these negative controls, we can compare how the Zipf's law exponent changes as cultural products are historically produced (Figure 3a-c). Compared to the subsampled control data sets of equal number of accumulated symbols, there is a significant departure of the exponent trajectory close to the beginning of the exponential growth phase (see SI for statistical analysis). This implies an increase in the ratio between imitation and innovation $(p / v)$, whether though plagiarism, standardization or lack of exploratory behaviour. This trend is reverted towards the end of the boom-and-bust cycle in Atari games and social media posts (Figure 3a,c), suggesting a change in the dynamics which can lead to a secondary wave of cultural diversity.

\section{High compressibility of cultural history with imitation}

Compressibility can be an adaptive feature of cultural transmission. Information is most efficiently transmitted when it is easier to learn and store in memory, e.g., during experiments of cultural transmission based on remembering items ${ }^{33}$. Repeated learning by new individuals makes cultural information increasingly compressible (or alternatively, decreasingly complex, see next section). For example, drawings become more schematic ${ }^{34}$, languages acquire regular structures ${ }^{12}$, and drumming patterns develop into musical rhythms ${ }^{35}$. Recently, compressible cultural patterns have emerged in an online collaborative art project involving over one million participants ${ }^{36}$. High density of digital art increased the competition among users, with more compressible artworks easier to reproduce and maintain than less predictable patterns. 
A consequence of positive feed-backs by widespread imitation is that artifacts tend to be more compressible (or simpler) than would otherwise be expected. Figure 3d-f shows the comparison of corpus compressibility in the three cultural domains and their negative controls (see Methods). Here, cultural compression decays exponentially as a function of accumulated symbol count. All cultural domains contain less information per bit than their controls, except in early Atari 2600 games (possibly due to the pressures of very small memory and tight performance constraints, see SI). This overall trend is consistently reinforced in the predicted region of collapse, with the two systems diverging shortly after the beginning of the boom-and-bust cycle. For sufficiently large timescales, corpus compressibility will tend to the theoretical entropy rate predicted for each cultural domain (see SI). In the long run, control data sets will be less predictable than cultural collapses whenever variability is preserved.

\section{Decline of cultural product complexity}

Complex knowledge has an intrinsic value because it is costly to invent and difficult to learn. Different measures of knowledge complexity have been discussed, but we are interested in detecting any complexity loss triggered by imitation and redundancy. The fingerprint of imitation can be found in structural properties of artifacts. First, knowledge can be generated from combinations of simpler components. For example, cultural products are assembled from components (byte codes or words) in mesoscale structures, whether machine instructions or sentences in natural language ${ }^{18}$. These components form bipartite networks $\left(B_{i}\right)$ where words are linked to the sentences in which they are present (see Methods). Each $B_{i}$ captures the internal structure of ideas and how they relate to each other for a given artifact. And second, not all combinations of components are equally useful, e.g., frequent combinations are simpler to obtain because lower information content. KolmogorovChaitin complexity $K(x)$ of the adjacency matrix of $B_{i}$, i.e. the size of the shortest program able to codify cultural structure (hereafter called cultural structural complexity, see Methods), provides the formal link between complexity and combinatorial frequency ${ }^{37,38}$. Kolmogorov complexity is uncomputable, but it can be approximated using the Block Decomposition Method $(\mathrm{BDM})^{13}$

In Fig. 3g-1 we show the evolution of the size normalized product complexity for different domains (NBDM), including our endogenous boom-and-bust and control data sets. For Atari (Fig. 3g) and Cryptocurrencies (Fig. 3h) a significant decrease in average product complexity is observed during peak cultural diversity, consistent with our predictions for increased imitation in a Polya urn generative process. This simplification does not occur in their respective controls, where no temporal trend is observed. In the case of r/PunPatrol (Fig. 3i), we found no significant changes in the time series, possibly due to the difficulty of approximating the Kolmogorov complexity of short sequences, highlighting the limitations of this metric on cultural products with a small number of components. However, $N B D M$ of the collated posts for any given period does display a marked downward trend in the r/PunPatrol data set while it remains constant in r/Politics (see SI). Although this coarse-graining precludes us from determining the significance of the observed pattern, it suggests that the reduction in complexity is indeed present in this example although difficult to observe in the smaller scales of individual social media posts. 


\section{Predicting Collapse}

Using the Polya urn generative process for culture production we can simulate boom-and-bust behaviour (see Methods) by incorporating the following assumptions: (1) An artifact's fitness depends on the presence of previously unobserved components; (2) the global urn exploration and reinforcement rates reflect changes in the population of creators and their strategies; (3) a coupling across the market population dynamics takes place, i.e. the artifact producer population grows or shrinks proportionally to the average product fitness and the current population size.

In Figure 4f-h we show fittings for the boom-and-bust model for each of our test data sets, as well as the average fitness predicted from our model $(\phi(t))$. Additionally, in Figure 4c-e we show how our hallmark metrics for imitation behave under different $p$ and $v$ values for a history of synthetic cultural products. Using a real Atari video game (1983 Pac-Man) as a template, we produce synthetic cultural histories using the same phrase-component sequence, and compute their zipf's law exponent, compressibility and complexity. As reported previously ${ }^{16}$, the observed symbol frequency rank exponent depends on the reinforcement and exploration rates with a power law relation of the form $f(R) \sim R^{-p / v}$, where $R$ stands for the rank of a given symbol. Artifact history compressibility shows an exponential decay towards the theoretical entropy production rate, which is high in diverse urns $(v>p)$ and low in urns dominated by imitation. In the case of product complexity, we observe a peak of structural complexity at intermediate values of imitation versus exploration. When there is an imbalance towards exploration $(v>p)$ the bipartite representation of cultural products is very sparse, composed of new components in every phrase which reduces their average size-normalized complexity. Conversely, for $p>>v$, there is a repetition of elements that drive the structural complexity down, implying a mechanistic link between exploratory balance and optimal complexity production rates in a Polya urn model of culture generation. In Figure $4 \mathrm{~b}$ we show the average amount of samplings required to find an element of the adjacent possible for different values of $p$ and $v$, supporting the claim that although innovation is possible in a system dominated by imitation, it becomes increasingly harder to create novel products when the common pool of ideas or technologies is saturated by the social transmission or reinforcement of old components.

\section{Avoiding collapse}

With the model proposed in the previous section we can also explore the limits of cultural collapse and what measures can be taken to avoid it. The first premise of the model sets some boundaries to the classes of systems in which our described endogenous collapse can occur. Systems or markets in which products have a large intrinsic fitness component will not require consistent innovation, and the recurrent sampling of the adjacent possible will not be a driving force in them. In Figure 5a we show that the boom-and-bust dynamics are preserved even if products have up to a $60 \%$ fitness contribution from other sources (see SI). Prior work has established the importance of innovation in the Atari video game system ${ }^{23}$, the cryptocurrency market $^{24,39}$ and popular memes in social media ${ }^{40,41}$, where a second release of a similar product (a video game clone, a cryptocurrency fork o a repost) is unlikely to have the same success as the original.

The second premise allows us to link a population structure of artifact producers to the overall Polya urn exploration and reinforcement rates. For the sake of simplicity we consider a two compartment population model $(x, y)$ which represent the 
two archetypal production strategies of innovation and imitation respectively (Figure 4a). In reality agents have a more fluid strategy that likely depends on their environment and likelihood of success ${ }^{42}$. To this end, we introduce some mutation rates between compartments, simulating their changes in strategies. We assume a linear relationship between the urn exploration and reinforcement parameters $(v, p)$ and the population sizes in each compartment, although these only need to be weakly coupled for the system to display endogenous collapse (Figure $5 b$ ).

In the final premise, we propose that a coupling between creators drives the boom-and-bust dynamics in these systems. In essence, current average fitness informs producers about future success, driving the growth or decay of the system as a whole. In this manner, success by a population of innovators can promote further investment and growth in the system, including the population of imitators. The model is robust to changes in this assumption, for even if innovators grow up to twice as fast as imitators the system still displays explosive growth followed by collapse (5c). Examples of this global coupling can be seen in all of our proposed endogenous collapse systems. For instance, the success of the Atari video game system led companies without expertise to the video game market, including the video game Pepsi invaders by Coca-cola Inc. (a clone of the highly successful Space Invaders) and Chase the wagon by Purina Inc. ${ }^{23}$. A similar case can be found in the cryptocurrency Kodakcoin, which was announced by Kodak Inc. as a way to capitalize on the crypto bubble ${ }^{43}$. In the case of subreddits, individual posts can recruit new users to the subreddit community, increasing the fitness or carrying capacity of the system. Subreddits compete for the attention of a common pool of users ${ }^{44}$, with meme producers migrating to other subreddits if they can better capitalize their posts in terms of votes and karma.

\section{Conclusions}

We have defined a theoretical, empirically-based framework for explaining innovation-driven cultural collapses. This is not a novel phenomena, but the study of cultural collapses has been hindered by the lack of a quantitative, widely accepted definition ${ }^{9}$. Understanding the growth and decay of diversity is a main question in both biology and social sciences ${ }^{45}$. Unlike biological innovation, which necessarily depends on a series of coordinated genetic mutations ${ }^{46}$, cultural innovation depends on a behavioral balance between frequency of reinforcing known components and addition of newer ones. Novelties are likely to be found when there is enough knowledge diversity, but diminishing returns in diversity can also take place when imitation (i.e., reinforcement of well-known components) is too strong. As we have shown, this can lead to premature convergence, followed by an eventual collapse into cultural uniformity and substantial knowledge losses. This suggests the endogenous origin of cultural collapse, which is not necessarily triggered by external events or environmental fluctuations.

Information technologies may increase the risk of cultural collapse. Digital and social media accelerated the changes to our social systems, with poorly understood consequences ${ }^{47}$. Imitation-driven collapses can be expected in technology and cultural domains facilitating the spread of imitation, like digital media ${ }^{48}$. Cultural agents carry out individual learning if it is adaptive or affordable, and switch to imitation otherwise, e.g., during the acquisition of complex knowledge it is beneficial to imitate from successful individual learners ${ }^{49}$. A typical example is software development. Programming a video game for the 
Atari 2600 is a time consuming task involving detailed knowledge of the hardware and extensive programming experience. Coexisting computer-based technologies like the Commodore Vic-20 mitigated this trend by providing intuitive and easy-to-use programming tools (see SI), and thus allowing social learning to generate reliable knowledge at a lower $\operatorname{cost}^{50}$.

To prevent maladaptive imitation and unnecessary accumulation of redundant artifacts, individuals should be able to adaptively detect and discard them ${ }^{51}$. For example, subreddits feature different levels of self-policing, which might preclude systemic hype and collapse with a more strict filter to the flood of non-novel artifacts (see SI for the community guidelines of our two studied subreddits). Filtering also emerged after the video game crash. When less innovative titles flooded the console market in the 1980s, Atari had no means for controlling them. In 1985, Nintendo imposed a quality-control process for any third-party developer, which had to submit their games for review and assurance ${ }^{23}$. Increasing transparency is also a pressing issue in our social media ecosystem ${ }^{47}$. For example, algorithmic biases can inadvertently promote high levels of conformity leading to cultural collapse $\mathrm{e}^{52}$.

Only reliable observations of cultural redundancy will hint at the risk of collapse. The hallmarks of imitation used in this study serve as early warning signals of an exploration-reinforcement imbalance. These indicators appear to be universal, and they can be easily implemented in existing databases. In this context, the framework presented here establishes a formal basis to study global dynamical patterns of innovation and traits of cultural systems, highlighting the dangers of conformity, imitation, standards and social norms to human endeavors ${ }^{47}$. While it provides a dire warning to novelty-driven markets, it also hints at the mechanisms required to mitigate innovation-driven cultural collapses.

\section{Methods}

\section{Data collection}

We downloaded a collection of video game ROM cartridges for the Atari 2600 (1977-1992) and Commodore Vic-20 (1980-1985) from the websites atarimania.com/index.html and www.tosecdev.org, respectively. We implemented a custom semi-automatic process to reverse-engineer the set of assembly source codes from the binary codes stored in these $N=738$ ROM files. Static code analyses cannot guarantee reliable source code reconstruction, and we need to augment the disassembly process with dynamic information about game playing (see SI for a full discussion of these computer systems and the reverseengineering process). Cryptocurrency white papers from the period 2009-2020 were collected from various online sources (whitepaperdatabase.com, allcryptowhitepapers.com, whitepaper.io) and dated according to their document production date when available or their first transaction otherwise (see SI). Bit strings were extracted from the pdf files using the Textract library for python. Scientific publications from the period 1980-2020 listed by PubMed with the keyword Optics were downloaded from their respective publication web resources. Pdf file processing was carried out as described for cryptocurrency white papers. Reddit posts were retrieved using the python reddit API wrapper (PRAW) from their respective subreddits (spanning approximately 330 days starting at Oct 18th 2018) by collating posts and comments using a depth-first strategy. All English bit strings were processed using the natural language toolkit for python (NLTK) and synsets were elaborated using the 
WordNet synonym sets by replacing each non stop-word with its most frequent synonym in the Brown corpus (see SI).

\section{Symbol frequency rank analysis}

For each test data set, accumulated cultural histories for varying amounts of total symbols were assembled (words in the case of english texts and machine code instructions in the case of video games). If the target amount of symbols required partitioning a cultural product, symbols were added to the cultural history from the beginning of the product until the desired length was reached. The Zipf's law exponent was then computed on the last 1.5 orders of magnitude of symbol frequency rank using the scipy curvefit function. 25 independent bootstraps of ordering within each period (years or ten day segments) were used to create a distribution of exponents.

\section{Compressibility}

In this work, we have considered the compressibility of accumulated cultural histories. Data compression algorithms can be used to measure the amount of information in different types of signals, such as audio, video or text. A data signal is compressible when there is a finite set of components (e.g., words from a known dictionary) contributing more information than the rest. Here, we used LZ77 text compression to decompose an ordered sequence (a string) of $L$ cultural symbols $S=\left(w_{1}, w_{2}, w_{3}, \ldots, w_{L}\right)$ (where $w_{i}$ is an instruction code or a text character) into distinct sub-sequences defining shortest prefixes that were not previously observed ${ }^{19}$. Each prefix was represented by the factor $(i, l)$, where $1 \leq i \leq L$ is the first index of the matched sub-sequence (or the symbol $w_{i}$ itself when observed for the first time) and $l \leq L$ is the number of symbols in the sub-sequence ${ }^{53}$. Computable information density (CID $)^{53}$ is proportional to the length of the compressed cultural history:

$$
C I D=\frac{L(S)}{L}
$$

where $L(S)$ is the total binary code length of the LZ77 compressed history (see SI). The compression factor (or compressibility) ${ }^{54}$ defines the amount of information per symbol of the uncompressed cultural history $S$ (measured in bits):

$$
\rho=\frac{C I D}{\log |\alpha|}
$$

where $\alpha$ is the alphabet of symbols used in the string $S$. Notice that CID is, in general, not the same as the compression factor, e.g., $0 \leq \rho \leq 1$ while $\mathrm{CID} \geq 0$ is unbounded. Information density and compressibility are equivalent for binary sequences, i.e., $|\alpha|=2$.

\section{Network representation of cultural artefacts}

The network representation decomposes a cultural product into the set of key components and removes lexical ambiguities (see SI). 
Given a specific problem, a simple solution is more easily stored and reliably transmitted than complex ones ${ }^{55}$. Cultural information is stored as recipes (algorithms) in the mind of individuals, and cognitive demands limits their diffusion. In this context, compressibility (see previous section) can detect statistical but not algorithmic regularities of cultural artifacts. For example, the string $0101010 . . .01$ displays high entropy (at the bit level) but it can be easily generated by a short computer program. Algorithmic complexity provides a robust measure for cultural randomness, and although uncomputable, it can be estimated by the Coding Theorem. The more frequent a string $x$ is, the lower its algorithmic complexity:

$$
K(x)=-\log m(x)+O(1)
$$

We consider a dynamical model defined by difference equations ${ }^{56}$, and thus taking place in discrete time. We numerically integrate the number of unused elements in the urn $(\mathscr{A}(t))$, also referred as the adjacent possible, as well as the number of components at least used once $(\mathscr{U}(t))$, which depend on the fraction of elements in the adjacent possible $(\Omega(t))$ :

$$
\begin{aligned}
& \frac{\mathscr{A}(t+\Delta t)-\mathscr{A}(t)}{\Delta t}=v(t) \Omega A(t) \\
& \frac{\mathscr{U}(t+\Delta t)-\mathscr{U}(t)}{\Delta t}=p(t)+\Omega(t)
\end{aligned}
$$

where $\Delta t$ is the observation interval, i.e., a year or 10-day period. Hereafter we will use notation $\Delta f(t)$ for the discrete-time difference equations for the data set appropriate observation interval. $\Omega(t)$ is defined as $\mathscr{A}(t) /(\mathscr{A}(t)+\mathscr{U}(t))$, and can be 
computed in order to calculate the probability of obtaining at least one novel item in a product of size $C$. Thus, $\Omega(t)$ becomes contingent on the historical values of $p(t)$ and $v(t)$ as well as the cultural productivity $N(t)$ and the artifact size $C$ (see SI):

$$
\Delta \Omega(t)=\frac{v(t) \Omega(t)}{C p(t) N(t)+(1+v(t)) \Omega(t)}
$$

where $C N(t)$ is the amount of sampling processes in the urn taking place in a given observation interval. In turn, $q(t)$ determines the average fitness of the generated cultural products $(\phi)$ :

$$
q(t)=1-(1-\Omega(t))^{C}
$$

. For simplicity, we consider a product novel if it contains at least one new element and assign it a fitness of +1 , while products without any novel element have a fitness of -1 . This, coupled with the system's inertia $(\mu)$ drives the discrete time evolution of cultural production $N(t)$, following:

$$
\Delta N(t)=\phi \mu N(t)=(2 q-1) \mu N(t)
$$

Finally, we incorporate a two-compartment population comprised of purely innovators and purely imitators, $x(t)$ and $y(t)$ respectively (Figure 4a). These populations of agents are in turn coupled to cultural productivity through $N(t)=x(t)+y(t)$, assuming for the sake of simplicity that each agent produces one artifact per unit of time. The agents in each compartment can switch strategies with some transition probabilities $\left(\delta_{x}, \delta_{y}\right.$ Figure $\left.4 a\right)$. The size of each population is directly linked to the urn $p(t)$ and $v(t)$ values, i.e. $p(t) / v(t)=y(t) / x(t)$.

$$
\Delta x(t)=\left[\mu(2 q-1)-\delta_{x}\right] x(t)+\delta_{y} y(t)
$$

$$
\Delta y(t)=\left[\mu(2 q-1)-\delta_{y}\right] y(t)+\delta_{x} x(t)
$$

. To simulate the evolutionary process, we set the switching probabilities $\delta_{x}$ and $\delta_{y}$, product size $C$, and initial time $\left(t_{0}\right)$. Starting from a population with a single innovator $x\left(t_{0}\right)=1$ and no imitators $y\left(t_{0}\right)=0$, we simulate the dynamics in discrete time $t_{0} \leq t<T$ using the previous set of equations. An evolutionary strategy (the covariance matrix adaptation evolution strategy, or CMA-ES, $\operatorname{see}^{57}$ ) was built to fit the temporal sequence of cultural productivity $N(t)$ (see SI). 


\section{References}

1. Lewis, H. M. \& Laland, K. N. Transmission fidelity is the key to the build-up of cumulative culture. Philos. Transactions Royal Soc. B: Biol. Sci. 367, 2171-2180 (2012).

2. Clay, Z. \& Tennie, C. Is overimitation a uniquely human phenomenon? insights from human children as compared to bonobos. Child Dev. 89, 1535-1544 (2018).

3. Singh, M. et al. Beyond social learning. Philos. Transactions Royal Soc. B: Biol. Sci. 376, 20200050, DOI: 10.1098/rstb. 2020.0050 (2021). https://royalsocietypublishing.org/doi/pdf/10.1098/rstb.2020.0050.

4. Henrich, J. The secret of our success: How culture is driving human evolution, domesticating our species, and making us smarter (Princeton University Press, 2017).

5. Kendal, R. L. et al. Social learning strategies: Bridge-building between fields. Trends cognitive sciences 22, 651-665 (2018).

6. Rogers, A. R. Does biology constrain culture? Am. Anthropol. 90, 819-831 (1988).

7. Enquist, M., Eriksson, K. \& Ghirlanda, S. Critical social learning: a solution to rogers's paradox of nonadaptive culture. Am. Anthropol. 109, 727-734 (2007).

8. Tchernichovski, O., Feher, O., Fimiarz, D. \& Conley, D. How social learning adds up to a culture: from birdsong to human public opinion. J. experimental biology 220, 124-132 (2017).

9. Zhang, H. \& Mace, R. Cultural extinction in evolutionary perspective. Evol. Hum. Sci. 3 (2021).

10. Tchernichovski, O., Eisenberg-Edidin, S. \& Jarvis, E. D. Balanced imitation sustains song culture in zebra finches. Nat. communications 12, 1-14 (2021).

11. Piantadosi, S. T. Zipf's word frequency law in natural language: A critical review and future directions. Psychon. bulletin \& review 21, 1112-1130 (2014).

12. Kirby, S., Tamariz, M., Cornish, H. \& Smith, K. Compression and communication in the cultural evolution of linguistic structure. Cognition 141, 87-102 (2015).

13. Zenil, H., Kiani, N. A., Zea, A. A. \& Tegnér, J. Causal deconvolution by algorithmic generative models. Nat. Mach. Intell. 1, 58-66 (2019).

14. Arthur, W. B. The nature of technology: What it is and how it evolves (Simon and Schuster, 2009).

15. Solé, R. \& Valverde, S. Evolving complexity: how tinkering shapes cells, software and ecological networks. Philos. Transactions Royal Soc. B 375, 20190325 (2020).

16. Tria, F., Loreto, V., Servedio, V. D. P. \& Strogatz, S. H. The dynamics of correlated novelties. Sci. reports 4, 1-8 (2014). 
17. Tria, F., Loreto, V. \& Servedio, V. D. Zipf's, heaps' and taylor's laws are determined by the expansion into the adjacent possible. Entropy 20, 752 (2018).

18. Buskell, A., Enquist, M. \& Jansson, F. A systems approach to cultural evolution. Palgrave Commun. 5, 1-15 (2019).

19. Ziv, J. \& Lempel, A. A universal algorithm for sequential data compression. IEEE Transactions on information theory $\mathbf{2 3}$, 337-343 (1977).

20. Lambert, B. et al. The pace of modern culture. Nat. human behaviour 4, 352-360 (2020).

21. Bentley, R. A. \& O'Brien, M. J. The acceleration of cultural change: From ancestors to algorithms (MIT Press, 2017).

22. Paich, M. \& Sterman, J. D. Boom, bust, and failures to learn in experimental markets. Manag. science 39, 1439-1458 (1993).

23. Montfort, N. \& Bogost, I. Racing the beam: The Atari video computer system (Mit Press, 2009).

24. ElBahrawy, A., Alessandretti, L., Kandler, A., Pastor-Satorras, R. \& Baronchelli, A. Evolutionary dynamics of the cryptocurrency market. Royal Soc. open science 4, 170623 (2017).

25. Tikhomirov, S. et al. Smartcheck: Static analysis of ethereum smart contracts. In Proceedings of the 1st International Workshop on Emerging Trends in Software Engineering for Blockchain, 9-16 (2018).

26. Liu, R., Wan, S., Zhang, Z. \& Zhao, X. Is the introduction of futures responsible for the crash of bitcoin? Finance Res. Lett. 34, 101259 (2020).

27. Valensise, C. M., Cinelli, M., Galeazzi, A. \& Quattrociocchi, W. Drifts and shifts: Characterizing the evolution of users interests on reddit. arXiv preprint arXiv:1912.09210 1 (2019).

28. Richterich, A. 'karma, precious karma!' karmawhoring on reddit and the front page's econometrisation. J. Peer Prod. 4 (2014).

29. Ferrer i Cancho, R. \& Solé, R. V. Two regimes in the frequency of words and the origins of complex lexicons: Zipf's law revisited. J. Quant. Linguist. 8, 165-173 (2001).

30. Gerlach, M. \& Altmann, E. G. Stochastic model for the vocabulary growth in natural languages. Phys. Rev. X 3, 021006 (2013).

31. Powers, D. M. Applications and explanations of zipf's law. In New methods in language processing and computational natural language learning (1998).

32. Font-Clos, F., Boleda, G. \& Corral, A. A scaling law beyond zipf's law and its relation to heaps' law. New J. Phys. 15, 093033 (2013).

33. Tamariz, M. \& Kirby, S. Culture: copying, compression, and conventionality. Cogn. science 39, 171-183 (2015).

34. Galantucci, B. An experimental study of the emergence of human communication systems. Cogn. science 29, 737-767 (2005). 
35. Ravignani, A., Delgado, T. \& Kirby, S. Musical evolution in the lab exhibits rhythmic universals. Nat. Hum. Behav. 1, 1-7 (2016).

36. Müller, T. F. \& Winters, J. Compression in cultural evolution: Homogeneity and structure in the emergence and evolution of a large-scale online collaborative art project. PloS one 13, e0202019 (2018).

37. Chaitin, G. J. On the length of programs for computing finite binary sequences. J. ACM (JACM) 13, 547-569 (1966).

38. Kolmogorov, A. N. On tables of random numbers. Theor. Comput. Sci. 207, 387-395 (1998).

39. Park, G., Shin, S. R. \& Choy, M. Early mover (dis) advantages and knowledge spillover effects on blockchain startups' funding and innovation performance. J. Bus. Res. 109, 64-75 (2020).

40. Coscia, M. Average is boring: How similarity kills a meme's success. Sci. reports 4, 1-7 (2014).

41. Coscia, M. Popularity spikes hurt future chances for viral propagation of protomemes. Commun. ACM 61, 70-77 (2017).

42. Miu, E., Gulley, N., Laland, K. N. \& Rendell, L. Flexible learning, rather than inveterate innovation or copying, drives cumulative knowledge gain. Sci. advances 6, eaaz0286 (2020).

43. Corbet, S., Larkin, C., Lucey, B. \& Yarovaya, L. Kodakcoin: a blockchain revolution or exploiting a potential cryptocurrency bubble? Appl. Econ. Lett. 27, 518-524 (2020).

44. Glenski, M., Pennycuff, C. \& Weninger, T. Consumers and curators: Browsing and voting patterns on reddit. IEEE Transactions on Comput. Soc. Syst. 4, 196-206 (2017).

45. Erwin, D. H. A conceptual framework of evolutionary novelty and innovation. Biol. Rev. 96, 1-15 (2021).

46. Wagner, G. P. \& Lynch, V. J. Evolutionary novelties. Curr. Biol. 20, R48-R52 (2010).

47. Bak-Coleman, J. B. et al. Stewardship of global collective behavior. Proc. Natl. Acad. Sci. 118 (2021).

48. Acerbi, A. Cultural evolution in the digital age (Oxford University Press, 2019).

49. Henrich, J. Demography and cultural evolution: how adaptive cultural processes can produce maladaptive losses: the tasmanian case. Am. antiquity 1, 197-214 (2004).

50. Boyd, R. \& Richerson, P. J. Why does culture increase human adaptability? Ethol. sociobiology 16, 125-143 (1995).

51. Enquist, M. \& Ghirlanda, S. Evolution of social learning does not explain the origin of human cumulative culture. $J$. theoretical biology 246, 129-135 (2007).

52. Clauset, A., Larremore, D. B. \& Sinatra, R. Data-driven predictions in the science of science. Science 355, 477-480 (2017).

53. Martiniani, S., Chaikin, P. M. \& Levine, D. Quantifying hidden order out of equilibrium. Phys. Rev. X 9, 011031 (2019).

54. Ziv, J. \& Lempel, A. Compression of individual sequences via variable-rate coding. IEEE Transactions on Inf. Theory 24, 530-536, DOI: 10.1109/TIT.1978.1055934 (1978). 
55. Winters, J. Escaping optimization traps: the role of cultural adaptation and cultural exaptation in facilitating open-ended cumulative dynamics. Palgrave Commun. 5, 1-13 (2019).

56. Yodzis, P. Introduction to theoretical ecology (HarperCollins College Division, 1989).

57. Hansen, N. The cma evolution strategy: a comparing review. Towards a new evolutionary computation 1, 75-102 (2006).

\section{Acknowledgements}

SDN would like to thank Matt Jackson for discussions on the cryptocurrency market. We thank Niles Eldredge, Alex Bentley, Mike O'Brien and Stuart A. Kauffman for useful discussions about the tempo and mode of cultural and technological evolution. We also thank Blai Vidiella and Josep Sardanyés for a careful review of the manuscript and helpful criticisms.

\section{Author contributions statement}

S.V. and S.D-N. conceived the research, S.V. and S.D-N. conducted the experiment, S.V. and S.D-N. analysed the results. All authors reviewed the manuscript. 

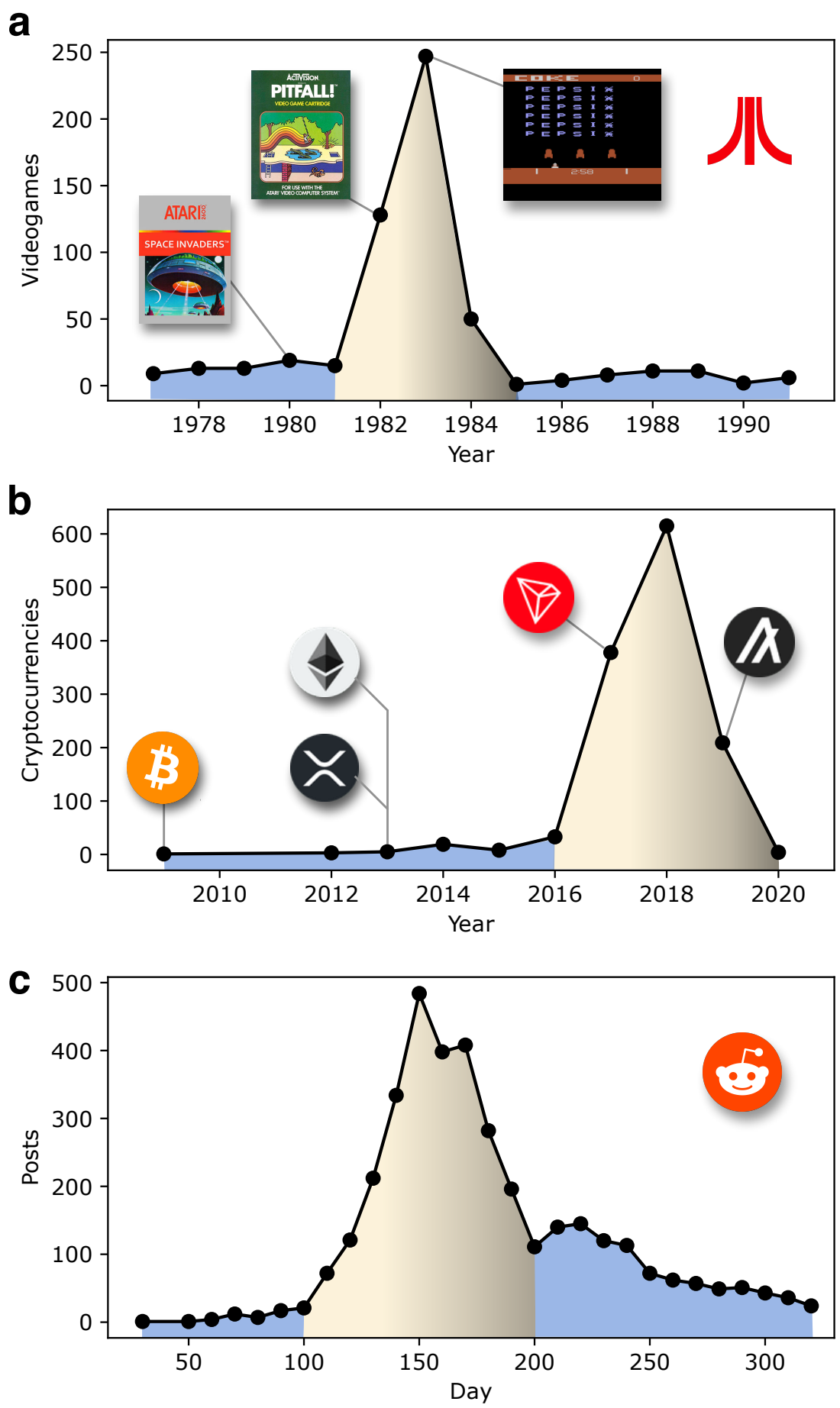

Figure 1. Cultural collapse in the dynamics of product diversity. The diversity of video games released for the Atari VCS (or Atari 2600) home console peaked in the year 1983 (a). The screenshots of several iconic games are highlighted, from highly successful games (Space Invaders, 1980) to original games (Pitfall!, 1982) to low-effort imitations, such as Pepsi Invaders (1983). Times series of the number of cryptocurrencies released between 2008 and 2020 (b). Key cryptocurrencies are shown as insets, including Bitcoin, Ripple, Ethereum, Tron and Algorand. Timeseries of post generation in a social community targeted toward meme and pun production on Reddit ( $r /$ PunPatrol) (c). See SI for a detailed discussion about these systems. 


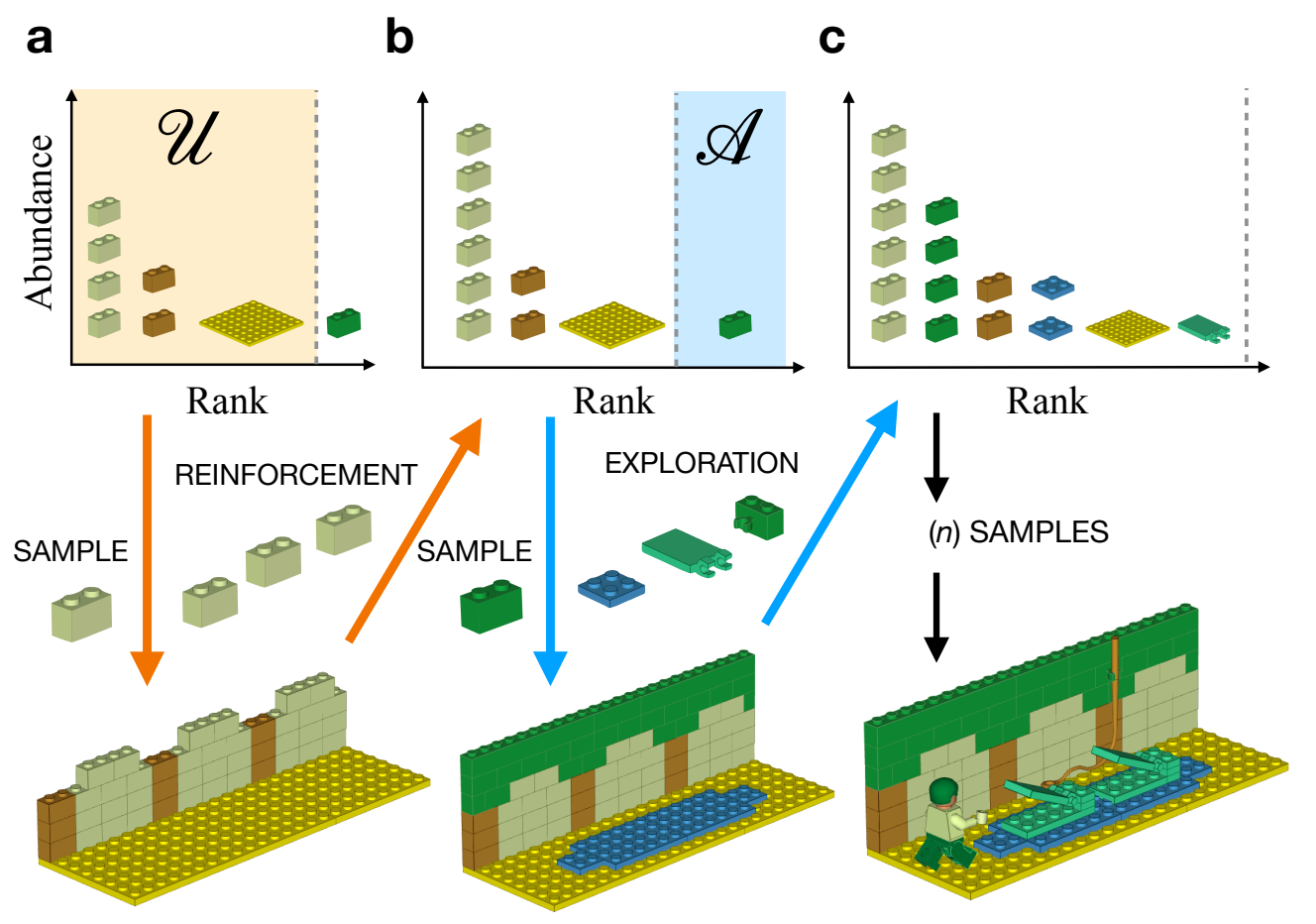

Figure 2. Polya's urn model of innovation. Cultural product (a complex Lego set, bottom) is progressively assembled by sampling a growing urn (e.g., a bag of Lego pieces, top). (a) Zipf's rank-abundance distribution on the top (i.e., the "urn") quantifies knowledge about components in one cultural domain (see text). At every step of this process, we sample one component from the urn. Depending on the abundance of this component, we apply two simple rules: when the component has been used previously $(\mathscr{U})$, we introduce $p$ copies of it into the urn, while (b) seeing the component for the first time $(\mathscr{A})$ triggers the addition of $v+1$ new components. (c) Complexity emerges when the balance between reinforcement and exploration allows consistent sampling of the adjacent possible $(\mathscr{A})$, which is a predicted outcome of cumulative culture (see text). 

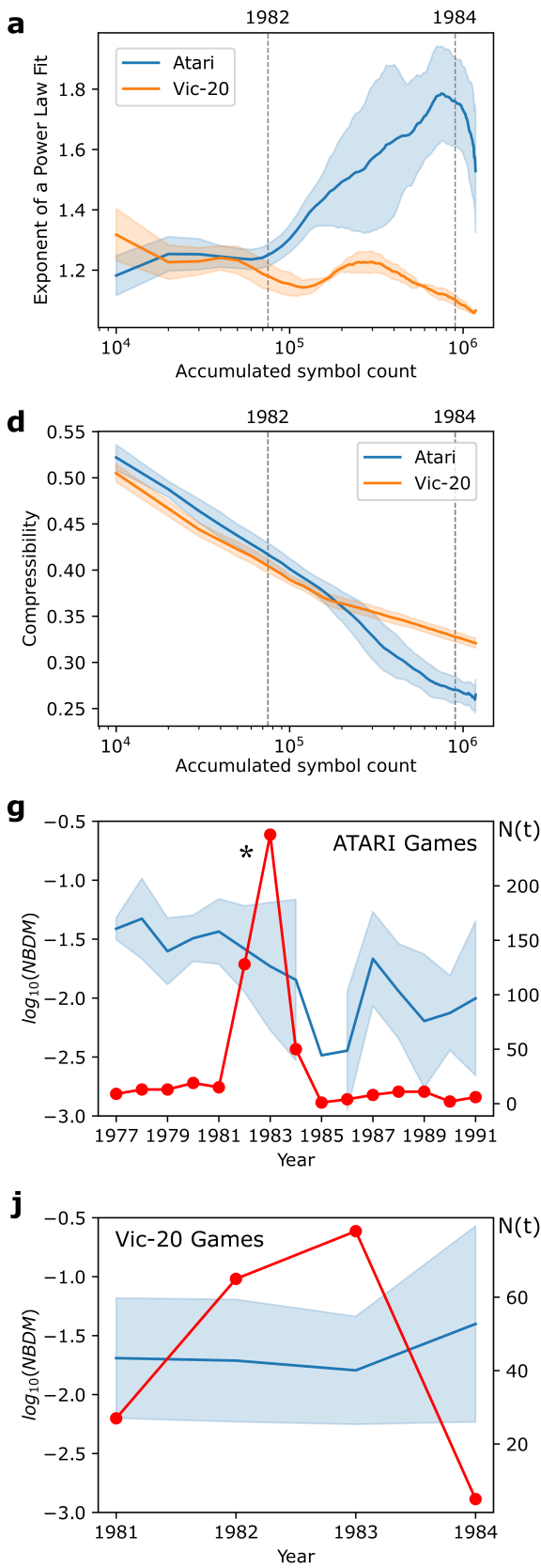
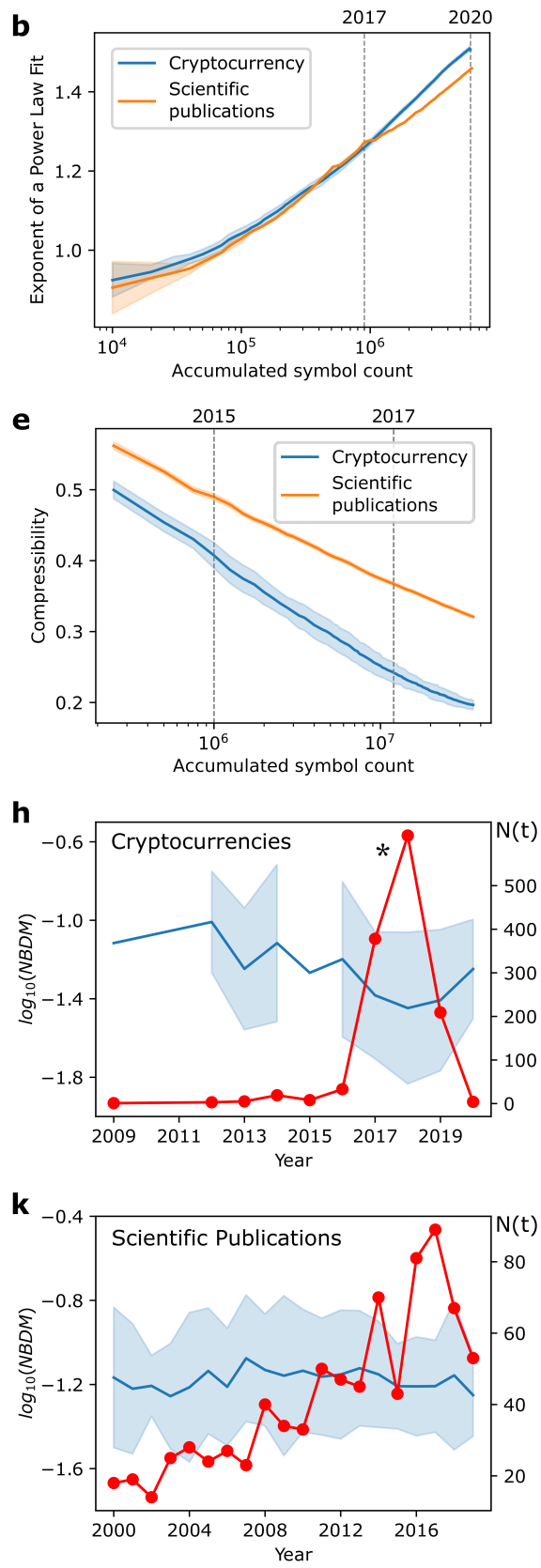
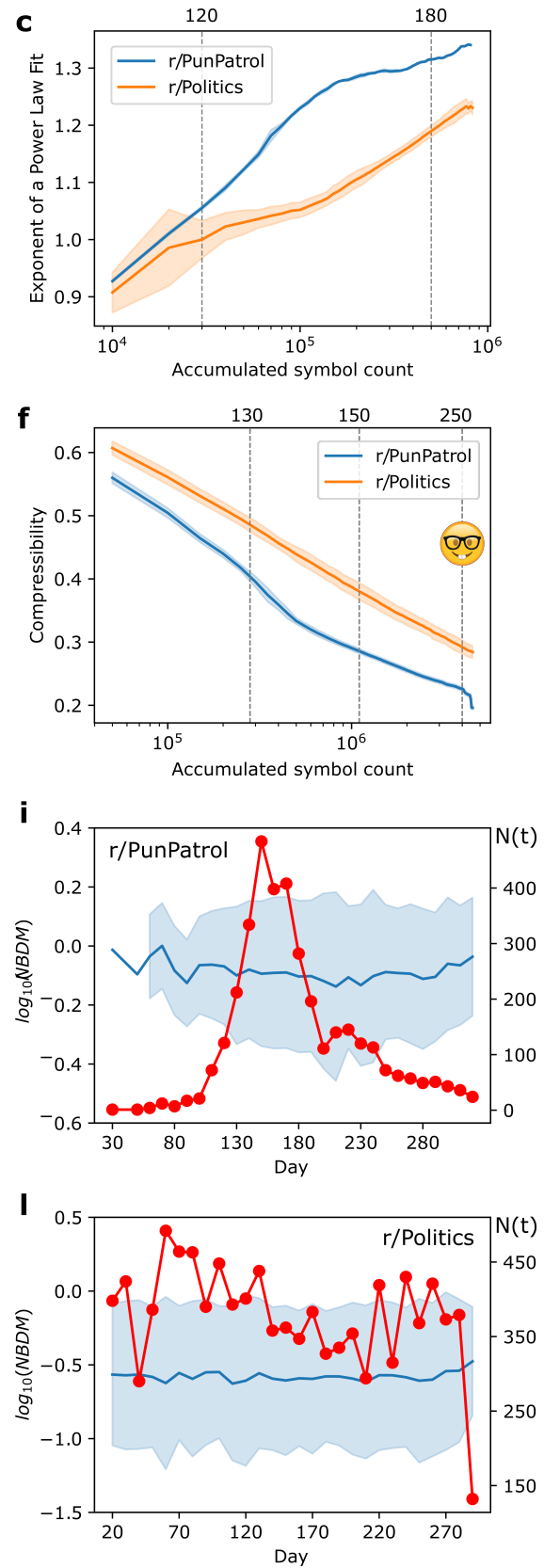

Figure 3. Hallmarks of imitation in the empirical boom-and-bust systems. Evolution of zipf's law exponent. For Atari and Vic-20 Games (a), cryptocurrencies and scientific publications (b), social network memes and political discussion (c), we show the exponent of a power law fit of the last 1.5 orders of magnitude of the frequency rank distribution for a given corpus size. Accumulated symbols are computer instructions for video games or words for English text, sequentially added to the cultural history according to their production date. The solid line represents the average exponent of 25 replicate sub-samplings and the shaded region encompasses one standard deviation of the distribution. As a guide, we also display vertical dashed lines marking the relevant dates for metric trend changes. Evolution of corpus compressibility. For Atari and Vic-20 Games (d), cryptocurrencies and scientific publications (e), social posts in the same two communities (f), we show the compressibility of the cumulative sequence of symbols (computer instructions or text characters). Corpus compressibility increases during in the boom-and-bust data sets due to lexical redundancies not found in their respective controls. The emoticon in (f) signals a sudden increase in the compressibility of $r$ /PunPatrol memes corresponding to the switch from lexical to graphical language. The solid line represents the average exponent of 25 replicate sub-samplings and the shaded region encompasses one standard deviation of the distribution. Evolution of cultural product complexity in different domains. We show the log transform of the size normalized artifact complexity (NBDM) for Atari Games (g), cryptocurrencies (h), social network memes (i), Vic-20 Games (j), scientific publications (k) and social network political discussion (l). The solid blue line represents the period (10 days or yearly) average product complexity and the shaded blue region encompasses one standard deviation of the distribution. For each domain we also show the cultural product diversity $(N(t))$ with a red line. 

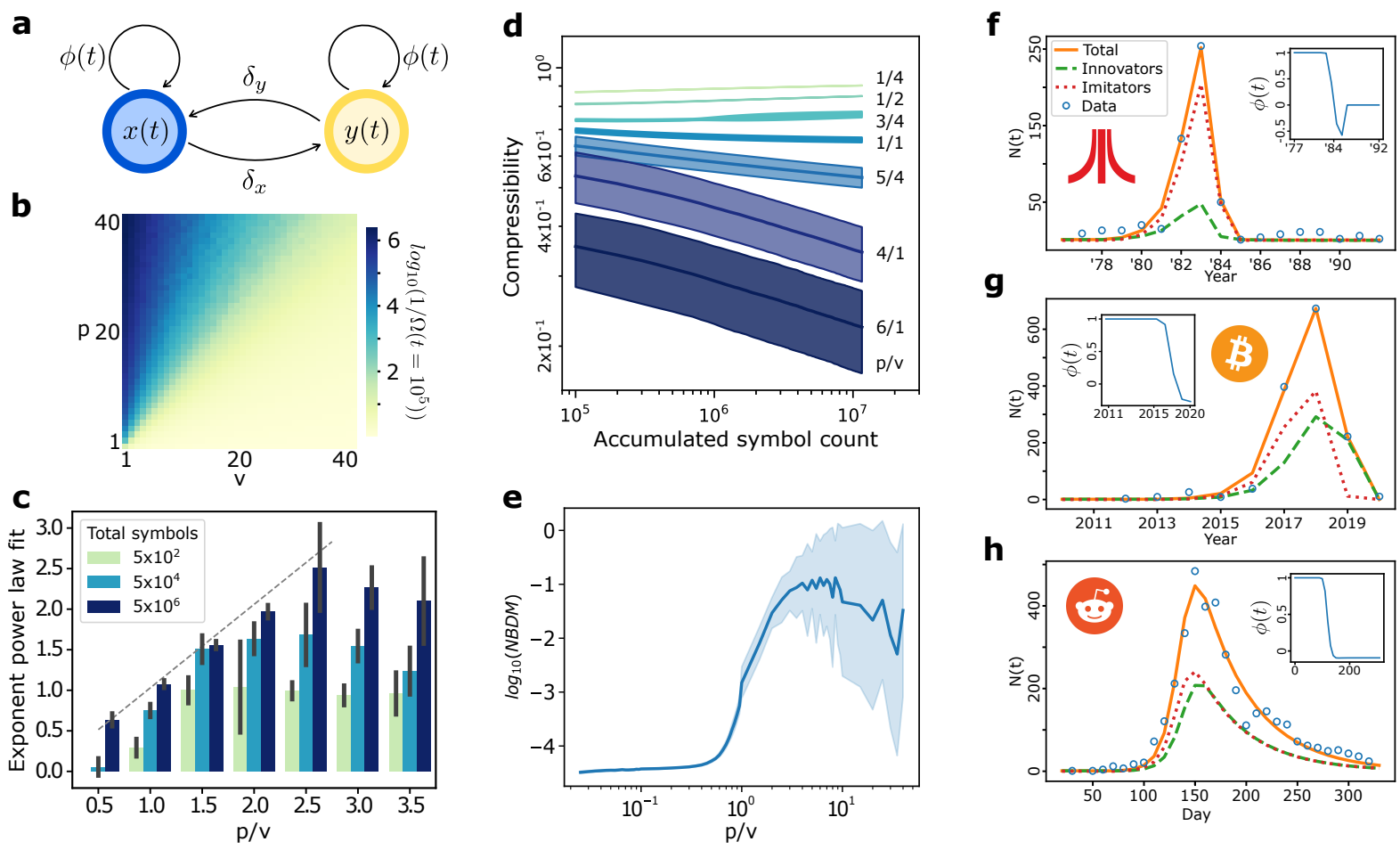

Figure 4. Modeling the boom-and-bust pattern and the hallmark traits of imitation in a synthetic cultural history. A population of innovators and imitators (a) is used as dynamic substrate for the urn exploration and reinforcement parameters. (b) For constant $p$ and $v$ values we show the expected time to find an unused component after $10^{5}$ samplings of the urn. (c) Component frequency in simulated histories follows a power law with an exponent close the urn internal distribution of components $p / v$. However, this reported exponent is dependent on the corpus size, in low total symbol corpuses the reported exponent is lower than the expected and it converges towards the bisector (grey dashed line marks) as more symbols are accumulated. Exponent calculated using the same method described previously, error bars represent one standard deviation of the sample. Data comprises 10 independent synthetic cultural histories using different values of the urn parameters and Pac-Man as a template. (d) Synthetic histories are more compressible when there is more imitation. For different values of $p$ and $v$ we show how the compressibility changes as more symbols are accumulated (see methods). Solid line represents the average compressibility of 10 independent synthetic cultural histories made of $10^{3}$ cultural products each, and the shaded region encompasses 1 standard deviation of the sample. (e) Average product complexity for different ratios of $p / v$. Although innovation can also reduce structural complexity, imitation produces simpler artifacts as well. Solid line marks the average normalized complexity for 10 replicates of each pair $p \in[1,40], v \in[1,40]$, plotted with logarithmic binning for the $p / v$ ratio. Shaded are represents 1 standard deviation of the sample. For Atari (f), Cryptocurrencies (g) and Reddit posts (h) we show the temporal dynamics predicted from our population dynamics model. We show the real cultural productivity during that period (open blue circles), the predicted cultural productivity (orange solid line) as well as the population of innovators and imitators at any given time (green and red dashed lines respectively). As an inset for each case, we report the average product fitness determined from to the product novelty in that period. 

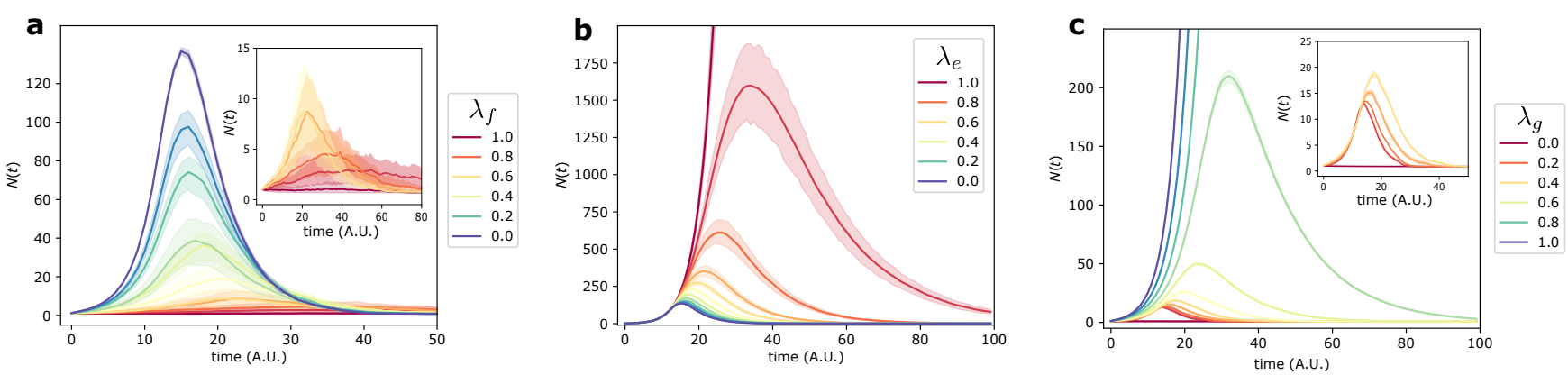

Figure 5. Avoiding cultural collapses. Effect of relaxing the model premises: the fraction of product fitness due to novelty (a), the connection between population structure and urn exploration parameters (b) and the coupled growth of innovators and imitators (c). In each case, we introduce a trade-off between the model mechanistic proposal and a random allocation of fitness $\left(\lambda_{f}\right)$, a random exploration of the urn $\left(\lambda_{e}\right)$ and a systematic impairment between the growth of the compartments $\left(\lambda_{g}\right)$. For each premise, there is a specific limit value upon which the system does not collapse. 


\title{
Supplementary Materials for "Imitation-driven Cultural Collapse"
}

\author{
Salva Duran-Nebreda and Sergi Valverde
}

May 2021

\section{Contents}

\begin{tabular}{lll}
\hline 1 & Introduction & 2
\end{tabular}

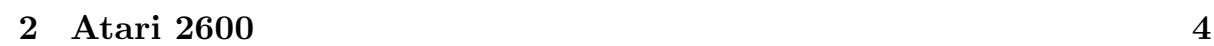

2.1 Atari Cartridges . . . . . . . . . . . . . . . 4

2.2 Attack of the clones $\ldots \ldots \ldots \ldots \ldots \ldots$

\begin{tabular}{lll}
\hline 3 & Commodore Vic-20 & 9
\end{tabular}

$3.1 \quad$ Vic-20 Cartridges . . . . . . . . . . . . . . . . . . . . 10

\begin{tabular}{|lll}
4 & Cryptocurrency & 14
\end{tabular}

4.1 The first wave . . . . . . . . . . . . . . . . . 15

$4.2 \quad$ An Ethereum-driven explosion $\ldots \ldots \ldots \ldots \ldots$

4.3 The crash of $2018 \ldots \ldots \ldots \ldots \ldots \ldots \ldots$

$\begin{array}{lll}5 & \text { Scientific publications dataset } & 19\end{array}$

$\begin{array}{lll}6 & \text { Reddit } & 20\end{array}$

6.1 Broadcasting cultural value . . . . . . . . . . . . . . . . . 21

6.2 A Meme factory . . . . . . . . . . . . . . . . . . . 21

6.3 Politics, PunPatrol and cultural collapse . . . . . . . . . . . 22

7 A combinatorial model of technological innovation 25

$7.1 \quad$ Parameter exploration of the premises of the model . . . . . . . . 27 
8 Expansion dynamics of the Adjacent Possible

8.1 Solution for $\bar{\Omega} \approx k t$. . . . . . . . . . . . . . . . . . . . 32

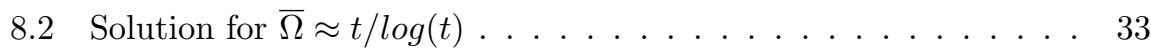

8.3 Solution for $\bar{\Omega} \approx t^{\alpha}$, where $0<\alpha<1$. . . . . . . . . . . . . 35

8.4 Solution for $\bar{\Omega} \approx k \ldots \ldots . \ldots \ldots 7$

8.5 Analytical derivation of $d \Omega / d t$. . . . . . . . . . . . . . . . 38

$9 \quad$ Lossless compression of cultural products 38

10 Bipartite network analysis of cultural products 41

11 Structural Complexity 43

11.1 Coarse-grained analysis of collated posts in the reddit data sets . 46

12 Statistical analysis of the hallmark metrics of imitation 48

12.1 Zipf's law exponents . . . . . . . . . . . . . . . . . . . . 48

12.2 Compressibility . . . . . . . . . . . . . . . . 49

12.3 Artifact complexity . . . . . . . . . . . . . . . 49

\section{Introduction}

In this document, we provide additional information about (1) data sets, (2) modeling approach, (3) parameter exploration of the assumptions of the model, (4) the metrics and methods used in the main text and (5) statistical analyses to validate the results.

Many empirical and theoretical studies have focused on how humans use social information. Outside controlled experimental conditions, it is much more difficult to obtain detailed (and complete) longitudinal records of cultural and technological evolution [1]. Data completeness affects many fields, and in particular archaeology suffers greatly from it. One way to deal with sampling limitations is to look at the population scale. For example, studies have tried to infer underlying mechanisms by modeling the evolution of artefact abundances under different assumptions (e.g., unbiased transmission, frequency-dependent copying). Here, we propose an alternative way to look at boom-and-boost dynamics in cultural evolution. As suggested in the main text, looking at time-series of 
cultural diversity is not sufficient to differentiate between endogenous and exogenous cultural collapses (see below). Instead, we need to analyze structural properties of artefacts at different levels.

To avoid these limitations, we decided to reconstruct 6 new large-scale datasets. These systems span years (and even decades) of cultural and technological evolution. To the best of our knowledge, none of these systems have been studied at the artifact genotype scale (there have been studies about Bitcoin but we are unaware of anyone performing textual analysis). In addition, our Bitcoin compilation includes rare cryptocurrency documents that are difficult to access today from products that have since become extinct. The present study represents one of the few attempts to reverse-engineer a large collection of Atari and Vic-20 video games (approximately 740 ROM files in total, see below). Reconstructing the source code of a video game from its machine code is a very time-consuming task, which requires expert and detailed knowledge about the underlying hardware. For this reason, previous works focused on the assembly codes of one (book) or a few video games (cite). Reverse-engineering classic video games at this scale is a technical challenge that requires developing semi-automatic methods (see Sections 2 and 3 below).

A key component of our study is the usage of empirical null models. First, models should be validated using multiple measurements (and not only one, e.g., frequency distributions). And second, it is very difficult to develop an accurate null model. As discussed above, data limitations often impede direct observation of transmission processes. Then, we can never ensure that a mathematical model includes all relevant mechanisms. Here, we take a different route: instead of solely relying on the proposed Polya Urn framework, we validate multiple observations of cultural collapse by taking the same set of measurements in another (comparable) empirical system that does not display an endogenous collapse. Interestingly, these reference systems can still display an exogenous collapse or follow different cultural productivity trends, yet our proposed metrics of imitation will be robust to temporal dynamics. For instance Vic-20 displays an exogenous collapse, scientific publications in optics follows an exponential growth trend and the number posts in $\mathbf{r} /$ Politics is largely constant for the period of analysis. 


\section{Atari 2600}

The Atari 2600 (originally branded Atari Video Computer System or Atari VCS) is a classic video game system, and the first successful programmable video game system (see Figure 11. The avalanche of game clones and modifications spurred by the commercial success of the coin-op game Pong (Atari, 1972), triggered Atari co-founder Nolan Bushnell to find ways to preserve Atari dominance in the early video game market. Coin-Op arcade games like Pong had the game logic pre-programmed in circuit boards [2]. Instead, the home system Atari 2600 attached a central processing unit (CPU), i.e., the inexpensive 8-bit microprocessor MOS Technology 6502, to an input/output chip capable of reading externally plug-gable memory banks, so-called ROM cartridges (see Figure1p).

\subsection{Atari Cartridges}

In this project, we reconstructed a database of $N=551$ video games released for the Atari 2600 home console. Each file in the database is an exact copy of the binary contents of the original ROM cartridge. This collection of Atari ROM cartridges were downloaded January, 18th, 2019 from the website http: //www.atarimania.com/index.html. The dataset includes almost every game released for the Atari 2600 between 1977 and 1992. Figure 2 displays several games for the Atari 2600. ROM cartridges have been an important component of home consoles and home computers (see below). Cartridges provide a rapid (almost instant) means to load the program and data required for playing a video game. Another important advantage of cartridge technology is the flexibility to use multiple games without changing the hardware 1

Game cartridges are more expensive that other storage systems (i.e., DVDs). The amount of information that could be stored in an Atari ROM cartridge was incredibly small for today's standards. From 1977 to 1979, the information contained in ROM cartridges was bound to $2 \mathrm{~K}$ (much less than a typical e-mail). During the booming period of the Atari 2600 market, cartridge size increased from $4 \mathrm{~K}$ to $8 \mathrm{~K}$. Later on, cartridges with $16 \mathrm{~K}, 24 \mathrm{~K}$ and $32 \mathrm{~K}$ of capacity were also possible. Programming home consoles was a true challenge due to the

\footnotetext{
${ }^{1}$ Modern game consoles like the Nintendo Switch also use cartridges
} 
a

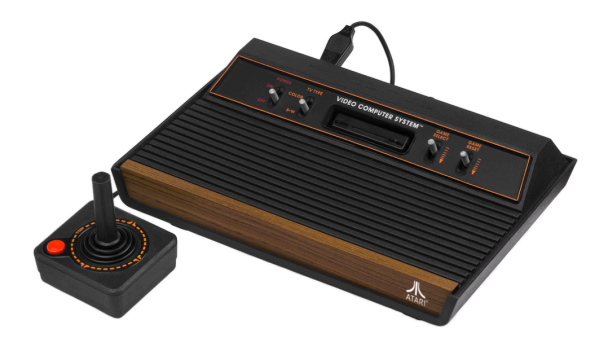

C

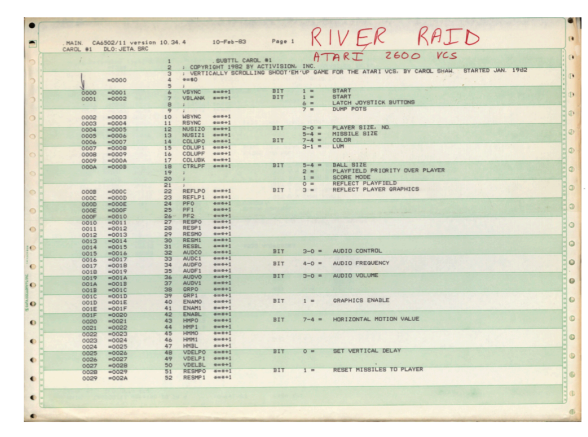

b

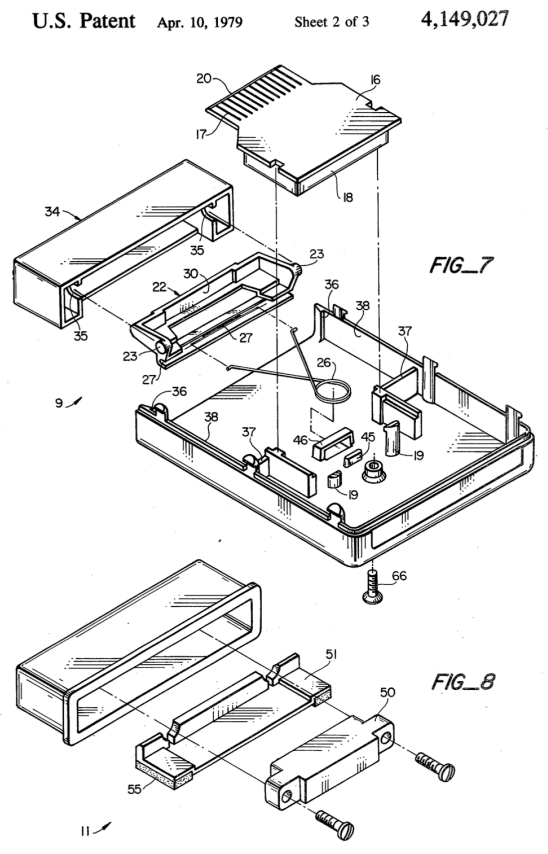

Figure 1: Modular (cartridge-based) architecture of the Atari VCS home console. (a) Four-switch Atari VCS model (released during 1980-1982) came with two joysticks and a slot used to insert game cartridges. (b) Figure 7 of the US patent 4149027 describing the internals of an Atari VCS game cartridge. A game cartridge is a plastic-encased board containing a memory ROM element (16) storing the program and data needed for playing a game. (c) The actual information stored in a ROM chip is a sequence of binary numbers (machine codes) encoding both game logic and data. Developing games codes is a difficult and time-consuming task that requires considerable expertise and skill. The panel shows the first page from Carol Shaw's printed code for the acclaimed River Raid videogame (Activision, 1983) (archives of The Strong museum).

serious constraints of the hardware (in particular when compared with the coinop arcade machines). Interestingly, this lack of proper hardware support forced programmers to innovate in software, and thus prolonging the life of the home console [3] (see below).

In this study, we retrace the cultural evolution of Atari 2600 video games by analysing the regularities and patterns of their machine codes. Unlike nat- 
ural text, the machine codes stored in ROM cartridge are not written using lexicographic symbols, but sequences of 8-bit codes meant to be read (and executed) by the CPU. During execution, the CPU decodes the instruction stored in a particular memory location and performs the corresponding action (e.g., changing the current sprite color, reading an input from the joystick or jumping to another memory location). Not all memory locations correspond to CPU instructions, some encode data values and/or parameters (e.g., a color value or a character definition).

Programmers wrote Atari games using a "high-level" programming language called assembly 4. Programs in assembler language are still difficult to understand, but are far easier to read than the binary codes of computer operations (which is the true language of computers). For example, the assembly instruction "LDA \#2" (tell the CPU to load the value 2 into the 'accumulator' register) represents a two-byte machine sequence "0xA9", "0x02" (codes are displayed in hexadecimal representation). In early computers, programmers had to translate assembly into machine code by hand. Fortunately, Atari programmers had a special program (so-called "assembler") to perform this translation automatically. Figure 1 shows the first page of Carol Shaw's printed assembly code for her popular game River Raid (Activision, 1983).

\subsection{Attack of the clones}

Our empirical and theoretical analysis suggests that an excess of imitation was one of the main drivers of the 1983 Atari market crash. The boom-and-bust dynamics has been described as a generic feature for new products [5, and the sales and profits of Atari is a common example discussed in the economics literature [6].

Many games released for the Atari 2600 were not truly original, but adaptations of coin-op arcade games (see Figure 2a) or licensed movies (see Figure 2;). Programmers could (almost) never build perfect copies of arcade games in the Atari 2600 due to (1) hardware limitations of the Atari 2600, (2) the different contexts of home and arcade play and (3) the different user interfaces [3]. In general, low-quality adaptations of arcade games had a negative reception (see Figure $2 \mathrm{~b})$. 
a

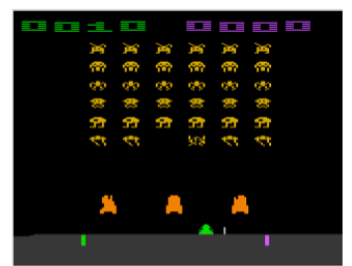

d

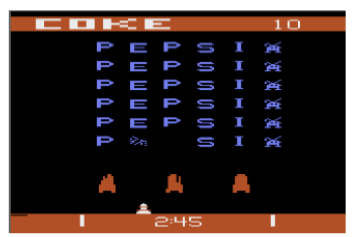

b

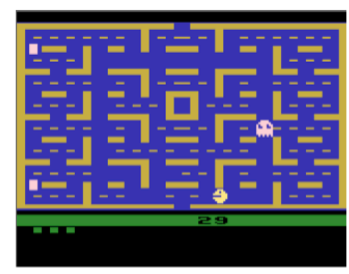

e

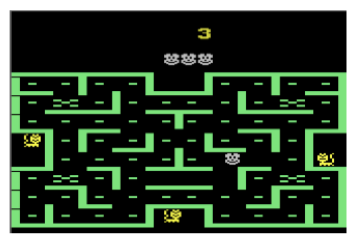

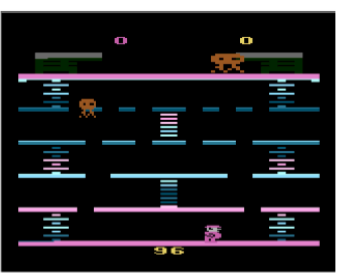

f

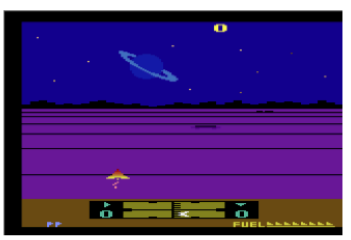

Figure 2: Screenshots of Atari 2600 games. (a) The diversity of Atari 2600 games exploded soon after the release of Space Invaders by Richard Maurer (Atari, 1980), an adaptation of the popular arcade game. (b) Pac-man by Tod Frye (Atari, 1982) was a top-selling game for the Atari 2600. It was claimed that Pac-Man technical limitations accelerated both the downfall of Atari and its 1983 market crash, (c) At the peak of the Atari market the quality of video games varied a lot. For example, Pac-Kong (Goliath, 1983) is a rare crossover between Pac-Man and Donkey Kong, (d) Pepsi-Invaders by Richard Maurer and Cristopher H. Omarzu was largely a clone of Space Invaders with different graphics, and only a very limited number of Omarzu cartridges were released (Atari, 1983), (e) Multiple clones of Pac-Man appeared during the boom of the Atari 2600 market, including Mouse Trap by Sylvia Day and Henry Will IV (Coleco, 1982), and (f) A prototype for a movie-inspired game ("The Last Starfighter") by Douglas Neubauer and Mimi Nyden (Atari, 1984) was released after the 1983 market crash under a different name, i.e., Solaris (Atari, 1986).

Sources of variation during social transmission can also generate innovations. For example, recognising the intrinsic limitations of the Atari 2600, Howard Scott Warshaw created the best-selling title Yar's Revenge (Atari, 1982). His game was a novel re-interpretation of Star Castle from Cinematronics (1980), a coin-op arcade using an advanced technology (vector graphics) that could not be matched by home consoles [3]. 
It is easy to find many examples of imitators of successful games, e.g., Space Invaders and Pac-man clones (see Figure 2e). Notice that imitation would not necessarily translate to exact clones (although in some cases this was the case, see Figure 2d). In general, simple measures of product similarity are poor predictors of cultural collapses (the diversity of cultural products peaks at the crash). A source of (trivial) game redundancy is re-releases of the same game under different titles/companies. In our dataset, we discarded all trivial clones and only analyzed a single copy (i.e., the first release) of every game. This gives additional support to our hypothesis, i.e. there was a clear increase of component redundancy at the booming of the Atari 2600 video game market.

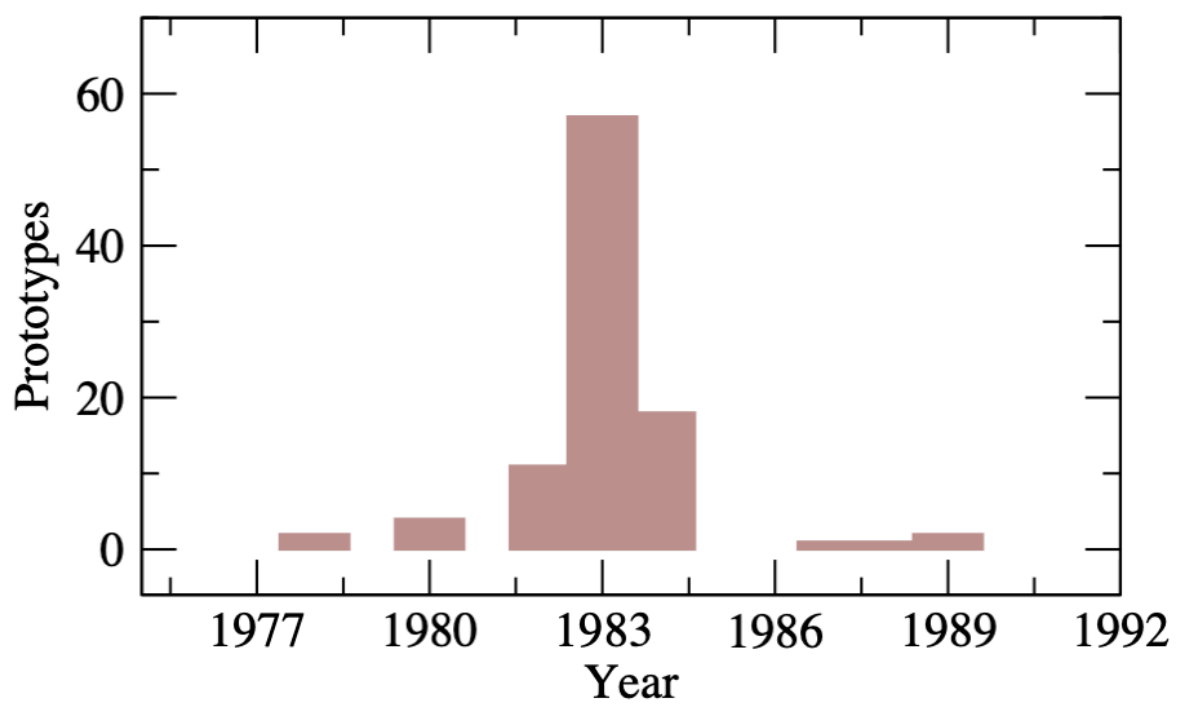

Figure 3: Time series of failed Atari 2600 games. Many games developed during the 1982-1984 period were never released (cancelled), postponed or released in very few cartridge numbers. This time series can be understood as a surrogate of economical losses and the drop in cultural fitness. Many third-party companies did not recover from their investments in developing (and releasing the corresponding cartridges for) these prototypes, and simply defaulted after the 1983 crash.

Even recombination has its own limits when it comes to the generation of novelties. In our model, the probability to obtain a novel combination depends on the abundance of single components in the urn. At the booming of Atari 
2600 , third-party developers attempted many combinations of elements from previously successful titles. One example is Pac-Kong by Goliath (1983), a game that can only be described as a weird mix between Pac-Man and Donkey Kong (see Figure 2).

As shown in the main text, changes in the urn reinforcement versus exploration balance $(p / v)$ can be interpreted as a signal for cultural collapse (which can be estimated through the exponent of the rank-abundance distribution). We have hypothesized that any obstacle preventing the reach of novelties yields a clear signal, i.e., a drop in cultural fitness. A close inspection of Atari 2600 video games suggest abundant anecdotal evidence for widespread imitation. Figure 3 shows the number of failed projects, that is, a significant number of game prototypes that were developed but never released (or in very few numbers, sometimes even less than 100 cartridges). As an example, consider the case when Atari acquired the rights to develop a game based on the blockbuster movie "The Last Starfighter" (see Figure 2f). The main character in the movie (Alex) played a game that was actually impossible to reproduce in any home console at that time (indeed, special F/X involved computer generated graphics). Again, Atari programmers were forced to innovate in order to overcome the strictures of the home console. Although the Atari 2600 version was finished in time for the movie premier, Atari ought to postpone the game release due to the market crash.

\section{Commodore Vic-20}

The Commodore Vic-20 (also known as the VC-20 in Germany and VIC-1001 in Japan) was the first computer of any kind to sell one million units. This lowcost personal computer was manufactured by Commodore during 1980-1985 and competed with other popular systems like the Apple II, the Radio Shack Color Computer and the Atari 400/800. Personal computers also competed with the home console market. These systems offered a video display system that included re-definable character shapes, superimposed movable objects (so-called "sprites") and high-resolution color graphics. In addition, personal computers incorporated a sound generator capable to play music and (with the addition 
of external hardware) even imitate human voices. Both the Atari 2600 and the Commodore Vic-20 coexisted in time and shared common features like: the same 8-bit microprocessor MOS Technology 6052, a small amount of RAM, and the possibility to use cartridges. On the other hand, home computers like the Vic-20 had some unique features that considerably expanded their range of applications beyond playing games (see Figure 44.

The temporal diversity of software products released for the Vic-20 is an example of "exogenous collapse". The boom-and-bust dynamics of Vic-20 closely follows the Atari crash (see Figure $3 \mathrm{j}$ in the main text). However, the decline of the Vic-20 was not a consequence of a lack of innovation (see below), but its eventual replacement by a more advanced model, i.e., the Commodore 64 (C64), released by the same company in 19832 Not only had the C64 more memory (64 Kb of RAM) but also vastly improved the sound and graphics capabilities of the Vic-20. The Vic-20 was discontinued in January, 1985 after selling more than 2 million units.

\subsection{Vic-20 Cartridges}

We reconstructed a database of $N=187$ cartridges released for the Commodore Vic-20 home computer in the period 1980-1985. We donwloaded all these game cartridges from the TOSEC Old School Emulation Center https: //www.tosecdev.org. Actually, many more VIC-20 games and applications $(\geq 1000)$ were developed in BASIC or in a mixture of both BASIC and assembly programming languages. Like many other home computers, the Vic-20 booted directly into the BASIC interpreter (see Figure 4). Commodore BASIC was much slower than the native machine code, but it was far more easier to understand. Interestingly, the ROM-resident BASIC interpreter and operating system core (so-called "KERNAL" 7]) provided useful subroutines reusable by games and applications, which eased software development.

To define a set of games comparable with the Atari dataset, here we only included all Vic-20 cartridges fully written in the assembly language. Games available in disk or cassette formats were also excluded. Because the Vic-20 and

\footnotetext{
${ }^{2}$ The Vic-20 was literally killed off in popularity by its manufacturer, Commodore, in an effort to redirect the market attention towards the improved Commodore 64 .
} 

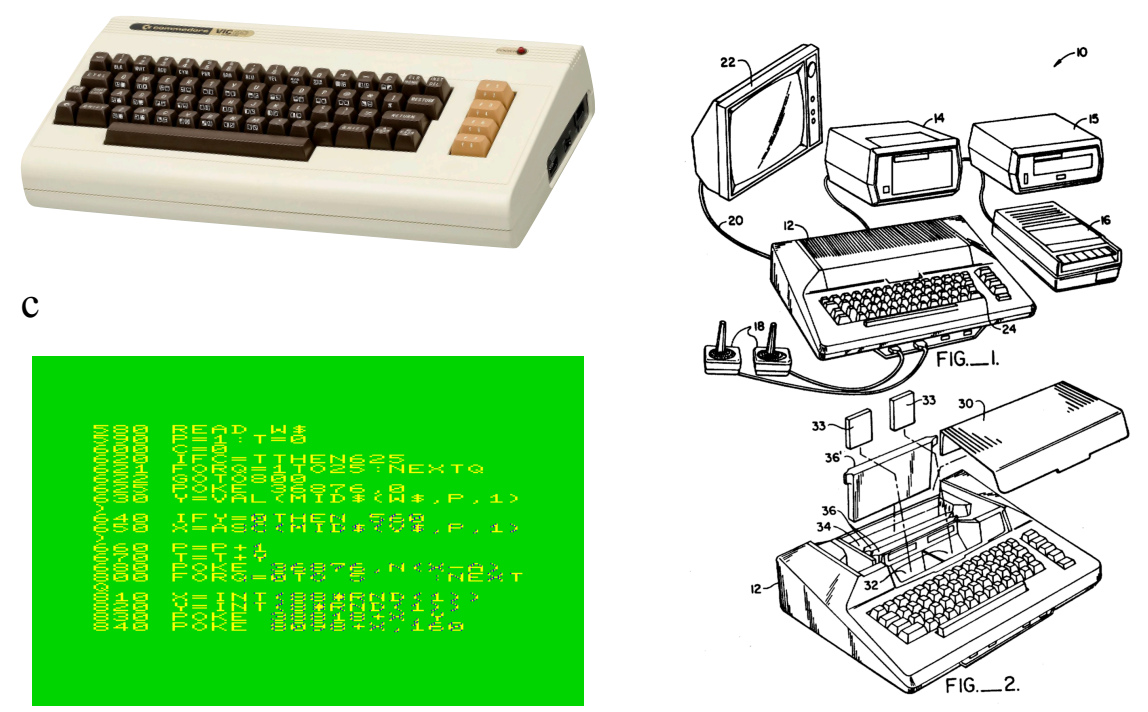

Figure 4: The personal computer introduced the general public to small, efficient data processing for home or business use. (a) The Commodore Vic-20 (released during 1980-1985) was a 8-bit home computer which came with a full keyboard and could be interfaced with many different peripherals, including one joystick, cartridges, and memory expansions. (b) Figure 2 of the US patent 4,471,465 displaying the Atari 400 home computer (12) in a typical configuration with a color TV (22), two joysticks (18), and external storage units (14, 15, 16). The top lid (30) of the computer could be removed to insert RAM and ROM cartridges (33) storing the program and data needed for a variety of a uses, including BASIC programming, word processing, and playing video games. (c) When the Commodore Vic-20 was started, the first screen the user could see was the prompt of the BASIC interpreter. Users entered BASIC commands with the keyboard to create, load, and execute complex programs like the one shown here.

Atari 2600 shared the same Motorola $6502 \mathrm{CPU}$, cartridges stored programs and data in the same machine (binary) language. Despite having similar CPUs, games for one machine could not be used in the another. For example, graphics support is very different in the two machines, e.g., the Vic-20 capabilities allowed 
a

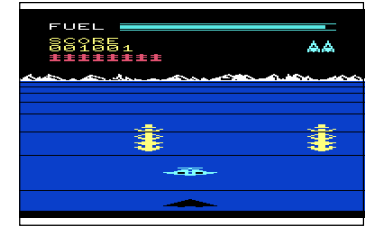

d

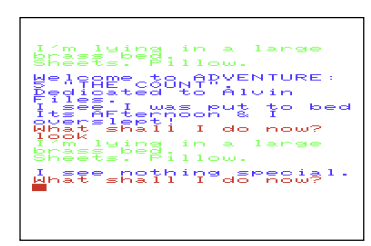

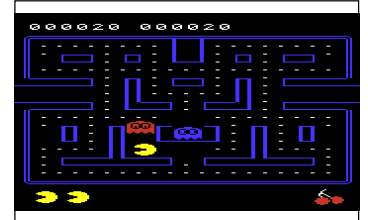

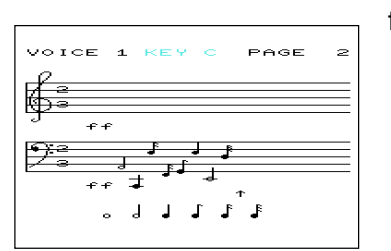

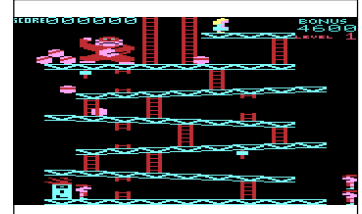

f

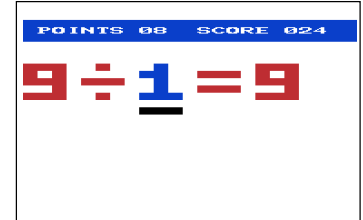

Figure 5: Screenshots of Vic-20 cartridges. We can see the repertory of Vic-20 applications includes not only clones of arcade games (a, b, c) but also original games exploiting the full range of different input/output peripherals, including: text adventures (d), music composition (e), and educational software (f).

larger graphics characters and faithful arcade conversions (e.g, compare the PacMan versions of Vic-20 and Atari 2600, see Figure 5b), while the Atari 2600 could use more colors and had better scrolling but stronger timing constraints 3.

In our study, we find signatures of collapse in the structural properties of cultural products. This involves analysing the original source codes for Atari and Vic-20 games. Unfortunately, we do not have access to the assembly code written by the programmers (except in a few cases 3 like Carol Shaw's River Raid, see Figure 17). Source code is discarded while generating the corresponding binary codes in the ROM cartridge (due to space constraints and copyright reasons). Theoretically, we could revert this process, but the task (known as "decompilation") is very expensive and involves a fair amount of human effort and expertise

It is clearly impractical to reverse engineer a large number of ROMs by hand. We designed (and implemented) a semi-automatic process to revert all

\footnotetext{
${ }^{3}$ Recently, the scans of the original source code for the Star Raiders video game (Atari, 1979) have re-surfaced

${ }^{4}$ Machine code decompilers for modern programming languages like Java and .NET often produce an almost perfect source code, and very close to the original sources, thanks to the metadata attached to the machine code.
} 
a

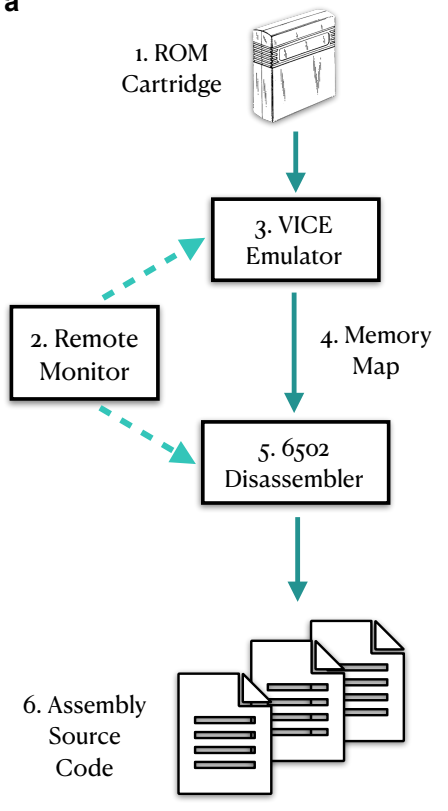

b
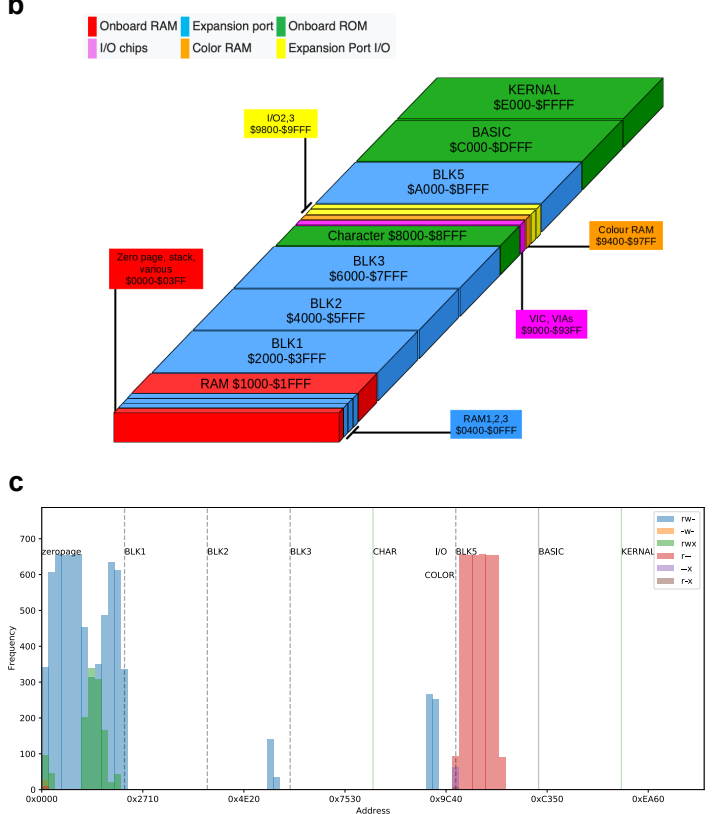

Figure 6: Reverse engineering a Vic-20 ROM cartridge. (a) Semi-automatic process of reconstructing the source assembly code from machine codes. This pipeline combines dynamic (runtime) information with static code analysis to obtain (a valid) source code version of the game in assembly language (see text for details). (b) Memory map of the Vic-20 indicates what memory locations (addresses) can be filled by the video game or belong to the system. This map determines the arrangement of the video game in the memory space. (c) Runtime memory map generated by the VICE emulator (see text) for the video game Arcadia (Imagine, 1982). The frequency of different types of memory accesses (read, write and/or execution) is displayed.

the binary codes in ROM cartridges to the closest possible version of the original assembly codes. The main issue for decompilation is figuring out whenever a memory location stores a machine instruction (and thus represents a piece of the full program) or not (and thus contains data like graphics, music or sounds). In addition, we need to determine the starting address of the game 5 , wich depends

\footnotetext{
${ }^{5}$ If a cartridge used the $\$ A 000$ memory block, it would automatically boot. Other cartridges (like Scott Adams' adventure ports) require the user to type the starting address at the BASIC prompt to start the game.
} 
on both the ROM size and the constraints defined by the Vic-20 memory map (see Figure 6p).

Figure 6 a depicts the different stages of our reverse-engineering process. The whole process is controlled from an external application, or monitor (2), that switches between two main processes. During the first stage, we play the game (1) using a popular open-source emulator for Commodore machines, or VICE (Versatile Commodore Emulator). This is one more accurate emulators for the Commodore Vic-20 and can be freely downloaded at https://vice-emu. sourceforge.io. VICE (3) keeps track of all memory accesses during gameplay (this feature must be enabled with the "--enable-cpuhistory" configure option). We modified the VICE emulator code to export the run-time map file (4), which classifies memory accesses as "read" (data), "read/write" (data) and "execution" (instructions) (see Figure 6e). In the second stage, a 6502 disassembler (5) reconstructs the (potential) source code in assembly language from the binary file of the ROM cartridge (1) and using the run-time map file (4) generated by the VICE emulator. Finally, we re-export the obtained assembly back into machine code (using the Dasm 8-bit assembler, available here https://dasm-assembler.github.io) to check the integrity of the reconstructed code. During this final validation step, we had to fix a small fraction of instructions by hand (less than $1 \%$ of generated instructions). Overall, we could generate a (syntactically correct) version of the assembly source code for all Vic-20 games under a month.

\section{Cryptocurrency}

Cryptocurrencies are decentralized payment networks that do not rely on traditional financial institutions. In order to function, they use sophisticated algorithms known as blockchains, used to validate users' transactions in a distributed register known as the ledger. The first cryptocurrency, Bitcoin, was released in 2009 by a person or group of people under the pseudonym "Satoshi Nakamoto" [8. The release of Bitcoin after the mortgage subprime crisis of 2008 is no coincidence, at this time there was wide interest in providing an easy to access digital currency that could exist unregulated outside the reach of national or 
international interests and financial institutions.

Bitcoin was designed to use a proof-of-work process: when validating groups of transactions (the blocks in the blockchain) a computationally costly algorithm needs to be completed [9], and in the process new bitcoins are awarded to the validators (also known as the miners). After someone has validated the block, it is added to the chain and broadcast through the network of miners, which accept it as ground truth for the next set of transactions. In the case of conflicting chains (due to simultaneous solutions or malicious attacks that broadcast tampered ledgers), the most frequently validated block by other miners is generally accepted. So in order to hack the Bitcoin ledger an attacker would need to control a majority of the computational power behind the currency.

Additionally, Bitcoin was designed with a baked-in deflationary scheme 10 that drives intense speculation in the cryptocurrency value. After specifically set dates, the Bitcoin payouts for validating blocks are halved, further driving the price of Bitcoin up as the supply does not scale with usage. Additionally, the rate at which solutions are found is always decreasing, requiring increasingly more computational power and energy to sustain blockchain operation and mine new Bitcoins. The operational costs of Bitcoin have garnered strong criticism from governments, environmentally concerned organizations and academia [9], since Bitcoin has an estimated global energy expenditure larger than some small countries [11, 12, 13, 14. It has also been suggested that if Bitcoin becomes globally adopted, it could push global warming estimates beyond $2^{\circ} \mathrm{C}$ with its mining associated emissions alone [15], with a massive carbon footprint associated to proof-of-work operation [16].

\subsection{The first wave}

Driven by the issues relating to energy costs, payout structure to the miners, governance of the community and algorithmic validation of operations, new cryptocurrencies emerged post 2009. For instance Peercoin was developed in 2012 with a proof-of-stake design: where block validation does not require costly computational tasks but instead consensus is created by the majority of coin holders at any given time. Despite a general trend of innovation, some of the currencies developed during this period were clones of Bitcoin with minimal 
changes to the core algorithm, sometimes only changing the name of the coin (e.g. Litecoin, Namecoin). These low-effort cryptocurrencies likely sought to capitalize on the Bitcoin brand value and the general crypto-illiteracy that was much more prevalent in those early years. During this period there were vast hikes in valuation that caught the general public interest and reinforced a positive trend in market value even if the birth rate of new cryptocurrencies did not skyrocket. Recent studies have shown that this initial period of crypto evolutionary dynamics could be understood as a neutral diffusion process in an unexplored technological landscape [17. Interestingly, after this first wave of duplicate coins, there is a measurable decrease in cultural productivity during the following years, perhaps hinting at later dynamics relating to the propagation of low-effort cultural artifacts [18.

\subsection{An Ethereum-driven explosion}

It was not until 2015 that the next step in crypto evolution took place: the release of Ethereum blockchain. Ethereum was truly innovative in that it lowered the entry barrier through the deployment of smart contracts and their ERC-20 tokens [19]. With these tools, people that did not have the technical knowledge to implement a cryptocurrency from scratch could perform Initial Coin Offerings (ICOs) and create an ecosystem of decentralized Apps hinging on smart contracts run on Ethereum blockchain (see an example use of ERC-20 tokens on the right most panel of figure $7 \mathrm{~b}$, representing the $\mathrm{W}$ Green Pay ecosystem). ICOs are the main way new cryptocurrencies acquire funds towards financing their own development by carrying out a pre-sale of their currency at a fixed and often discounted price. This easiness to find funds and run a blockchain promoted an explosion of coins in the following years, for instance in 2017 the amount of new cryptocurrencies was five times the accumulated diversity until that point. These new cryptocurrencies were not necessarily tied to technological improvements, but to new business areas that were not explored before: agriculture, healthcare, finance, real estate development, gambling, etc.

Some of these coins extensively overlap in target audience and do not offer any technological advancements as they make use of Ethereum technology for their implementation and launch. Their project leaders were not mathemati- 
cians or computer scientists, but people with an economics background that found the opportunity to create new financial instruments using cryptocurrency now that the technical entry barrier was lowered. Some of them were using their cryptocurrencies as a form of ponzi scheme, vanishing with millions of dollars as their users requested the promised features or milestones 20 .

\subsection{The crash of 2018}

This situation leads us to the sudden devaluation that happened in 2018 and the cultural collapse in terms of artifact production that followed. In 2018 the crypto market bubble burst, with Bitcoin losing $80 \%$ of its value during that year (from $\sim 20.000 \$$ to $\sim 4.000 \$$ ). This protracted economic downturn has been traditionally linked to the systemic overvaluation of the assets, as well as the listing of Bitcoin futures in traditional markets like the Chicago Mercantile Exchange 21. This meant that predictions on Bitcoin future value could be leveraged for financial gain, and once enough bad predictions were made the system entered a negative feedback loop of asset depreciation. In the following years, new cryptocurrencies were released at a much more contained pace, reaching an astounding 20 fold decrease in 2020 compared to 2018. As of 2021, there has been substantial economic recovery for the main players in the market, Bitcoin reaching currently $\sim 50.000 \$$. However, many cryptocurrencies are still below pre-crash valuation and many have since become extinct.

While the market crash will surely be of interest and modeled for years to come, here we are not concerned with the dynamics of asset valuation but with the tempo of cultural production, i.e. the rate at which new cryptocurrencies are produced and how these cultural artifacts relate to each other as an explanatory factor. In order to compare these artifacts we make use of their "white papers" (figure 7). These are technical and financial documents written in natural language that outline the project milestones as well as the technological specifications of each cryptocurrency and the expected usage that the creators envision for their technology. Usually several pages long, white papers include sections regarding algorithmic functioning, financing aspects of the project, partnerships with other major companies in the sector, etc. whatever the creators think is necessary to get new users on board with the currency. 


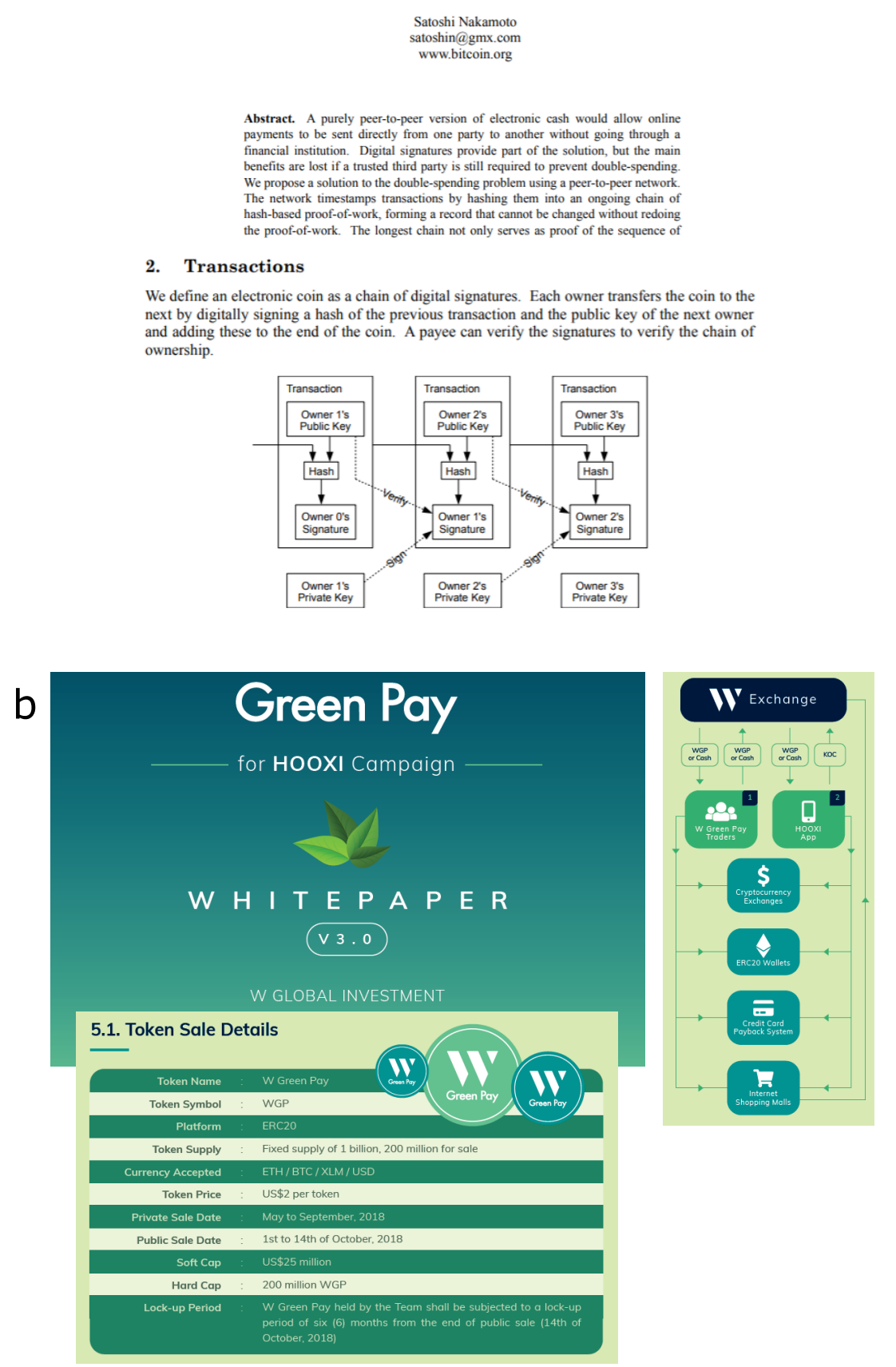

Figure 7: Examples of cryptocurrency white papers. (a) Bitcoin white paper (2009) is a technical/scientific document targeted at a computer science audience. (b) W Green Pay white paper (2018) is a much more accessible document with content mostly targeted to investors and users. 
These documents, typically in pdf form, were retrieved on the 15th of March 2020 from several sources $[22,23,24,25$. Our data records are surely an incomplete representation of the actual cultural process, as many projects fail and many are simply not documented. White papers written in languages other than english or made of collections of images that could not be translated to bit strings through the python Textract library [26] (version 1.6.3) were discarded. The final list of documents $(N=1275)$ were timestamped with the document production date if it was available, such dates can sometimes precede launch dates if white papers are released as a engagement or dissemination tools before the cryptocurrency is usable. If the document production date was not available, we used the Initial Coin Offering date as retrieved on March 15th from ICO's holder [27] and ICO's Rating 28] web resources. If no ICO took place or no information about the ICO remains, the first registered transaction of the coin was used instead, as obtained from their economic data in [29, 30].

\section{$5 \quad$ Scientific publications dataset}

Given that cryptocurrency white papers are presented in a scientific format and often deal with technical innovations in the area of computer science, we used a set of scientific publications as the negative control of innovation in a similar format and language. Scientific publications can have some variations in their format due to journal-specific structure, yet often require a review of the state of the art in the particular knowledge domain, clear innovation with respect to prior published articles and domain specific articulation of ideas or jargon. This features make them comparable to the cryptocurrency white papers, which also often include a brief history of cryptocurrencies and blockchain algorithms, computer science and financial jargon and in theory should include differentiating traits with respect to prior cryptocurrencies, i.e. a certain degree of innovation as captured in their components and structure.

Additionally, in order to provide a fair comparison, we chose to create a database with a given keyword as to constrain the cultural component spread. If publications from all fields were allowed within the dataset, that would likely increase the reported innovation of the negative control, amplifying the trends 
and divergences reported in the main text results. However, this would be a unfair negative control, since (following our generative framework of cumulative culture) publications from widely different disciplines are unlikely to be created from the same set of components. Instead, distinct scientific disciplines are likely to have their own respective pools of ideas and component technologies, with some cross-fertilization due to interdisciplinary research.

In this negative control with increased innovation, we assembled a list of publication DOIs from the field of optics between the years 1980 and 2020, as reported by the PubMed search engine [31. From this initial list, some articles were removed if they could not be retrieved due to a paywall, were not written in English or if the Textract library ([26], version 1.6.3) discussed previously did not accurately process the text from the pdf files. Finally, we assembled a collection of $N=1273$ files, spread across the time period reported previously but mostly concentrated in the 2000-2020 era. Interestingly, scientific publications in this knowledge domain in particular seem to be exponentially increasing according to PubMed.

\section{Reddit}

Reddit is a social network branded as a news aggregator and content discussion forum founded in June of 2005, about the same time as other giants of the sector such as facebook and twitter. Users in Reddit are typically anonymous and post content that they find relevant, whether locally hosted or located elsewhere in the web, and discuss it with other users in topic-focused forums called subreddits. Any registered user can create a new subreddit, in order to engage with other "redditors" that might be interested in a new topic or to promote a different kind of discussion for a topic that already exists (i.e. with differently aligned political views or restrictions on language or content). Policing of content happens through moderators, these are "superusers" with the privilege to remove posts and comments, ban users from posting in the subreddit (in a temporal or permanent fashion) and lock posts so that no more discussion can happen, among other things. This can happen due to reddit-wide content infringement policies or more specific subreddit guidelines, but it does 
not always pertain itself with content imitation or "reposting" as it is known by reddit users. Indeed, successful content is frequently posted again by the same or other users.

\subsection{Broadcasting cultural value}

Posts and comments can receive up votes and down votes from other users (only one vote per user) and are casted to signal whether they find it relevant or agreeable (upvotes and downvotes cancel each other to create an overall score, see figure 8 a). This voting mechanism typically results in rich-gets-richer dynamics in terms of score distribution, with few posts and users amassing most of the upvotes, given that users do not tend to read all the comments in a post or all the posts in a subreddit or even the content they are upvoting or downvoting 32. The standard settings of reddit visualization promote newer and higherscore content over older and less upvoted posts within a subreddit, making it gain more views and potentially more upvotes $[33$. For more in depth analysis on the topic, the subreddits $\mathbf{r}$ /TheoryOfReddit and $\mathbf{r}$ /dataisbeautiful have meta discussions about the statistics of their own functioning.

At the same time, account-wide accumulated score (called "karma") is regarded as the overall relevance and status of a user. However, it is still debated whether post created by a high-status user have significantly better chances at garnering upvotes and support, since redditors do not typically check user "karma" before considering voting on a post or a comment. While this score reinforcement process has its downsides, reddit users believe that it ensures a more democratic filter of the commercial news cycle. In particular, the use of account-wide scores has been linked to the production of less innovative and controversial cultural products or posts 34, instead signalling the production of content able to be quickly consumed and appeal to a mass social media audience.

\subsection{A Meme factory}

Some subreddits exhibit well thought, lengthy posts and discussions, while others are specifically targeted towards the production of controversial or niche content. However, most of reddit's posts are short comments [35] consisting of 
puns and "memes" [36. Content format and length has great impact in the consumption and upvoting dynamics: readily digestible content such as short videos, memes and puns usually garner more attention. Some subreddits are notoriously geared towards the production of memes and low-effort posts, this appears to be the default state of culture production in subreddits where there is no strong content policing and amass certain popularity. As more memetic products are generated, subreddits might also be able to recruit more new users, the average user lifetime in a given subreddit (conceptually the user attention span) being quite short ( $\sim 30$ days [35]). And as more new users are recruited less quality posts are produced, geared towards the more general audience the subreddit has acquired. This process can lead to runaway effects or tipping points in subreddit dynamics: population size has a positive feedback loop with itself through the production of more easily consumed posts and propagating memes.

\subsection{Politics, PunPatrol and cultural collapse}

This brings us to the topic cultural dynamics in subreddits, how communities grow and collapse while producing complex cultural artifacts in the form of posts and comments. Here we have taken the main post text as well as the post comments as a single cultural product. This is supported by the fact that most comments refer to the main post or contain culturally adjacent content. There is even a subreddit called $\mathbf{r}$ /TheRealJoke were pun refinement in the comments of a post are highlighted. In this study we have analyzed the cultural dynamics of two subreddits: $\mathbf{r} /$ Politics and $\mathbf{r} / \mathbf{P u n P a t r o l}$. The first one corresponds to an extensively moderated community (figure 8p), with strong content policing and long, non-memetic posts and discussions relating to current political events in the United States of America. This has sometimes elicited strong criticism on the moderation of the subreddit, calling it an echo chamber of political ideas. However, r/Politics has historically displayed a stable community and acts as a negative control for our framework of imitation-driven collapse. Conversely, r/PunPatrol features less stringent posting rules (figure 8p) and contains subreddit shibboleths and memetic post as its most frequent content. Additionally, $\mathbf{r} /$ PunPatrol has displayed interesting temporal features shown in figure $1 \mathrm{c}$ of 

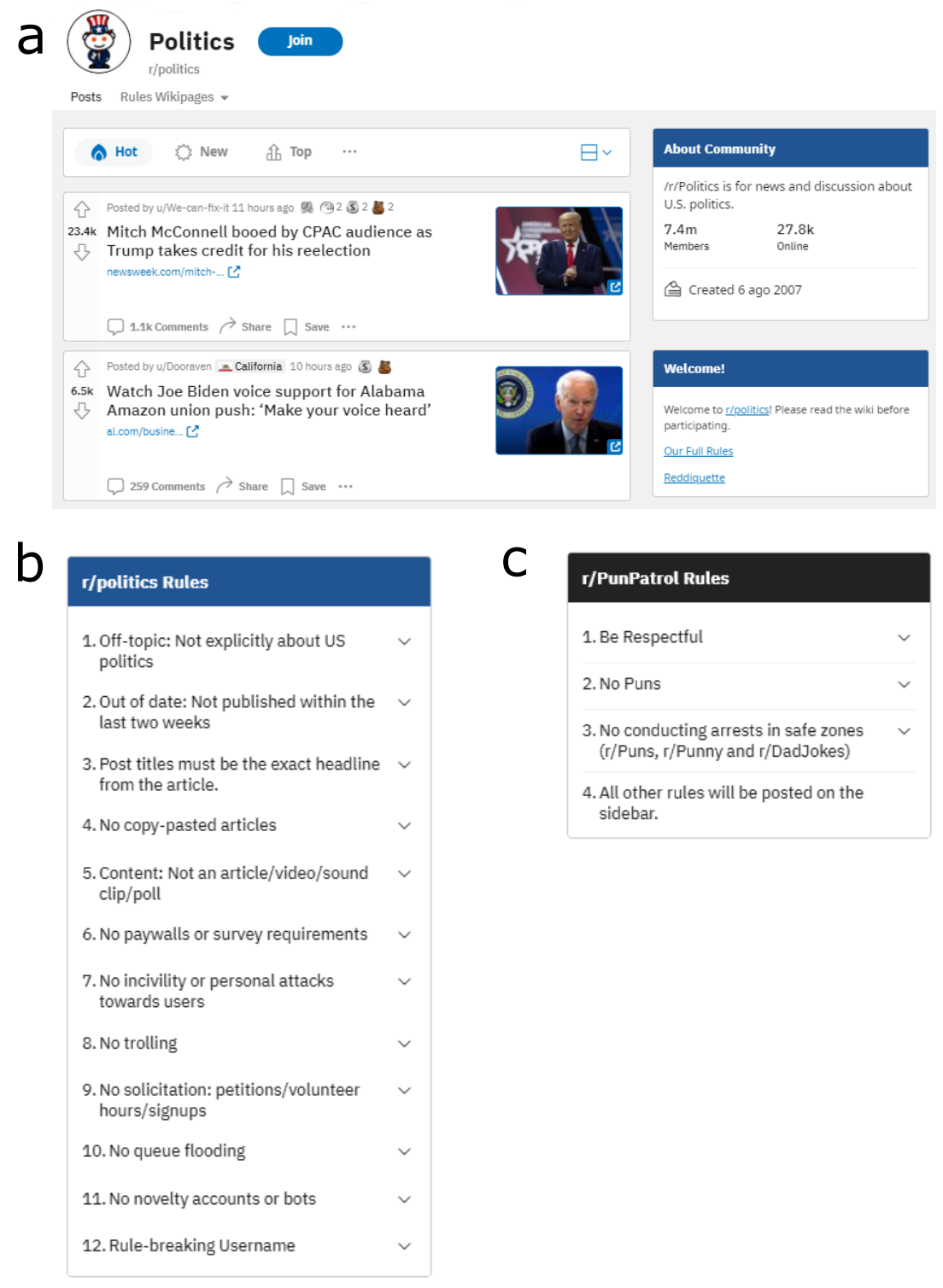
r/PunPatrol Rules
1. Be Respectful
2. No Puns
3. No conducting arrests in safe zones ( $r /$ Puns, $r /$ Punny and $r /$ DadJokes)
4. All other rules will be posted on the sidebar.

Figure 8: Reddit community rules allow for different cultural dynamics. (a) View of the typical subreddit layout in which users interact to produce and vote on content relevance. (b) Self imposed rules in $\mathbf{r} /$ Politics include strong policing on low-effort content while $\mathbf{r} / \mathbf{P u n P a t r o l}$ rules are much less stringent (c). 

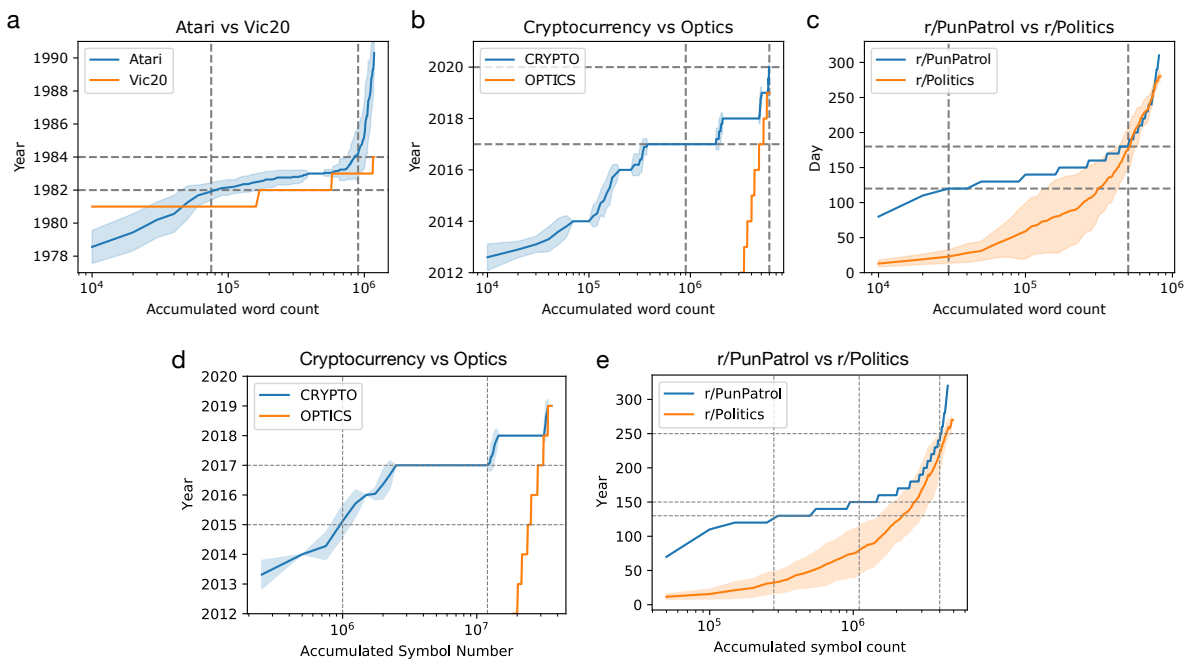

Figure 9: Cumulative growth of total Atari code instructions (a), cryptocurrency white paper words (b), post words in $\mathbf{r} /$ PunPatrol (c), cryptocurrency white paper symbols (d), and post symbols in $\mathbf{r} /$ PunPatrol (e). For each domain we also include the equivalent data in their respective negative controls. The larger dataset of the two was subsampled across the whole time period in order to obtain a comparable amount of total words. The solid line represents the average year in 25 independent subsamplings, and the shaded area encompasses one standard deviation of the sample.

the main text, namely rapid growth in artifact productivity followed by a sudden collapse in the number of post in the subreddit on the timescale of weeks. We downloaded both of the subreddits posts in the relevant time periods using the python wrapper for the reddit API (PRAW) [37, collating the post main text and its comments using a depth-first recursive strategy. This amounted to 10999 posts for $\mathbf{r} /$ Politics and 10761 posts for $\mathbf{r} /$ PunPatrol. Using this time-stamped cultural corpus as a starting point, we then processed the texts following the WordNet Synset strategy (see Section 10). 


\section{A combinatorial model of technological inno- vation}

In this section we describe the general Polya Urn algorithm used in this study, following 38. Below is the pseudocode implementation of an Urn with reinforcement and cultural generation of artifacts of a given size. For a cultural history $X$ of size $H$ products, each composed of $C$ elements we carry out:

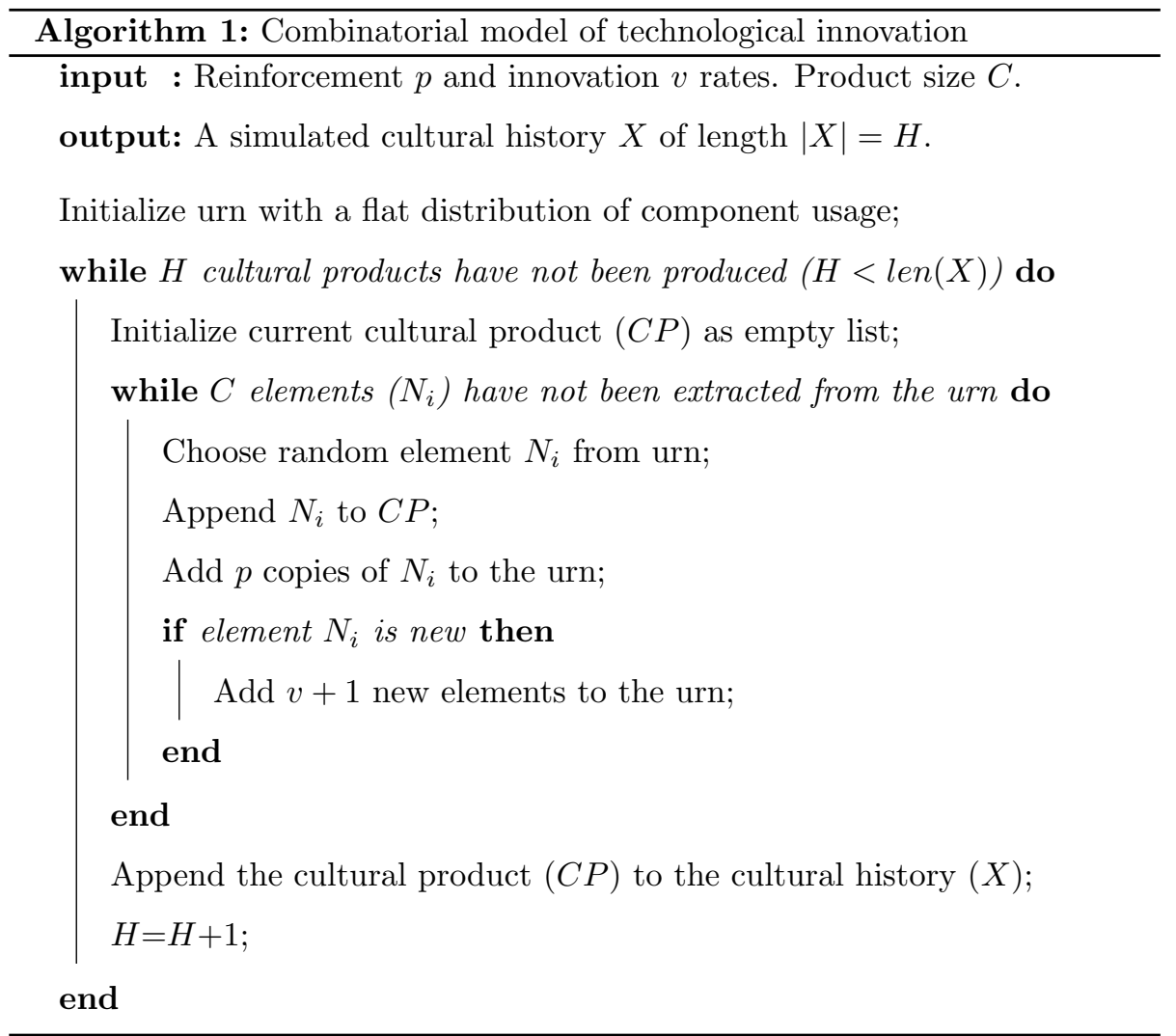

Additionally, we adapted this general algorithm in order to simulate and fit the real time series of Atari, Cryptocurrency and Reddit. At its core, it makes use of the predictions obtained from mathematical analysis of the adjacent possible in the Polya Urn (see Section 8).

Previous models have considered the rate of exploration of the adjacent possible $(v)$ and the rate of reinforcement $(p)$ as external, fixed parameters and/or intrinsically determined. This assumes the frequency of learning is stable along the cumulative cultural process. However, the tempo and mode and cultural evolution reflects how the community preferences for reinforcement and explo- 
ration change in time.

Although real culture producers will likely exist in a continuum of strategies for artifact generation [39, we make the necessary simplification that real cultural dynamics can be modeled by the behaviour of two archetypal and opposed strategies. Let's divide the population of agents creating cultural artefacts into two compartments $x$ and $y$ representing individual learners (i.e., those agents that tend to expand the adjacent possible) and social learners (i.e., reinforces of the already used cultural components), respectively. Each agent generates one artefact of size $C$ per unit of time, so $x(t)+y(t)$ would then represent the total amount of cultural artefacts generated at any given time $N(t)$ as well as the population of agents at $(t)$. The average fitness $\Phi$ is a function of the probability $\Omega(t)$ of generating novelties (i.e., a product is novel if uses one new component at least). This dynamical population model is thus centered around the strategies of the artifact producers instead of other recent approaches that focus on the relation between technologies themselves [40].

We simulate the population dynamics of innovators and imitators coupled with the process of creating a cultural history with the following pseudo-code, 
based on a simplified two-compartment Polya Urn:

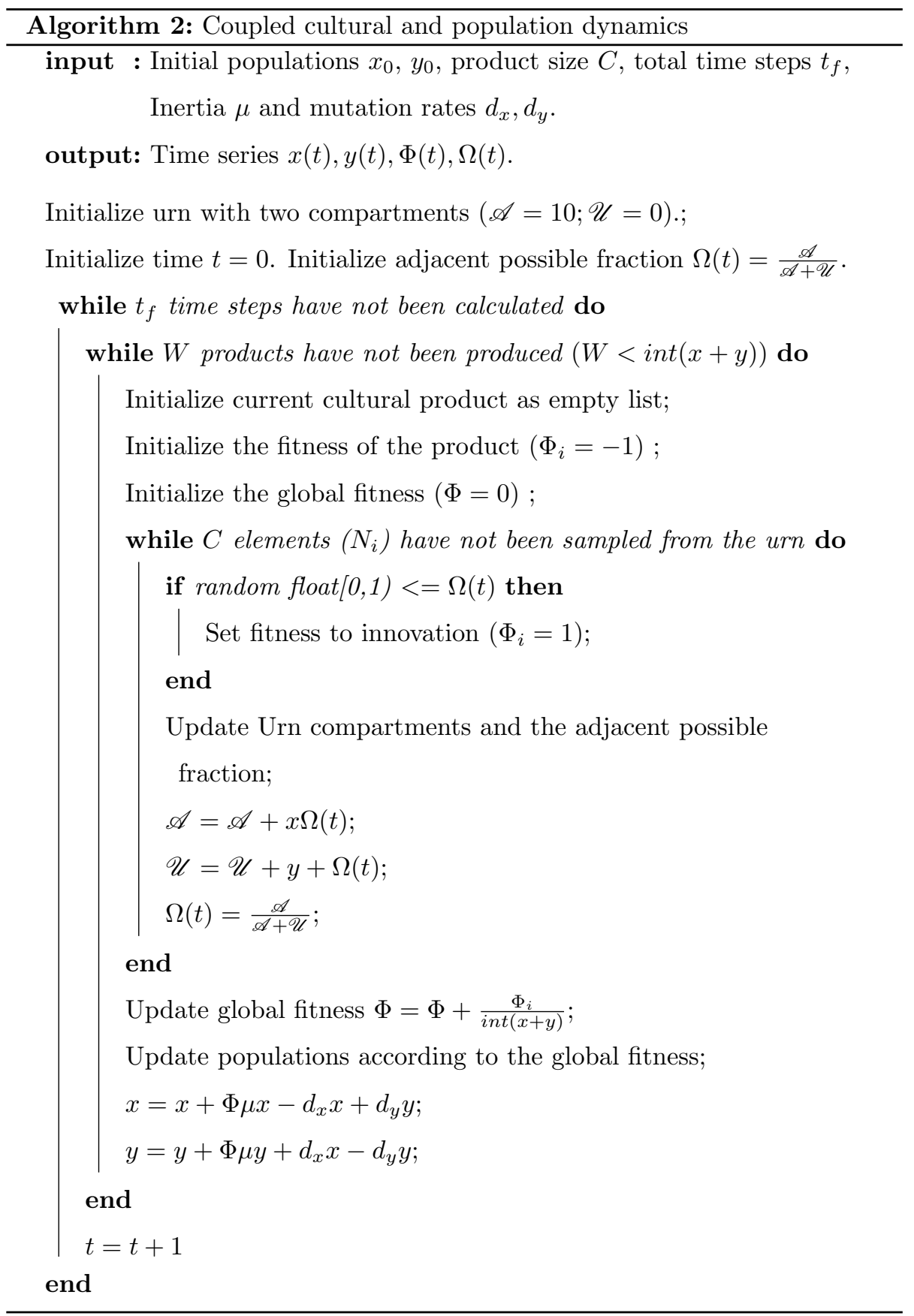

\subsection{Parameter exploration of the premises of the model}

In this section we will show the robustness of the model to the assumptions described in the main text. These are: (1) An artifact's fitness depends on the 


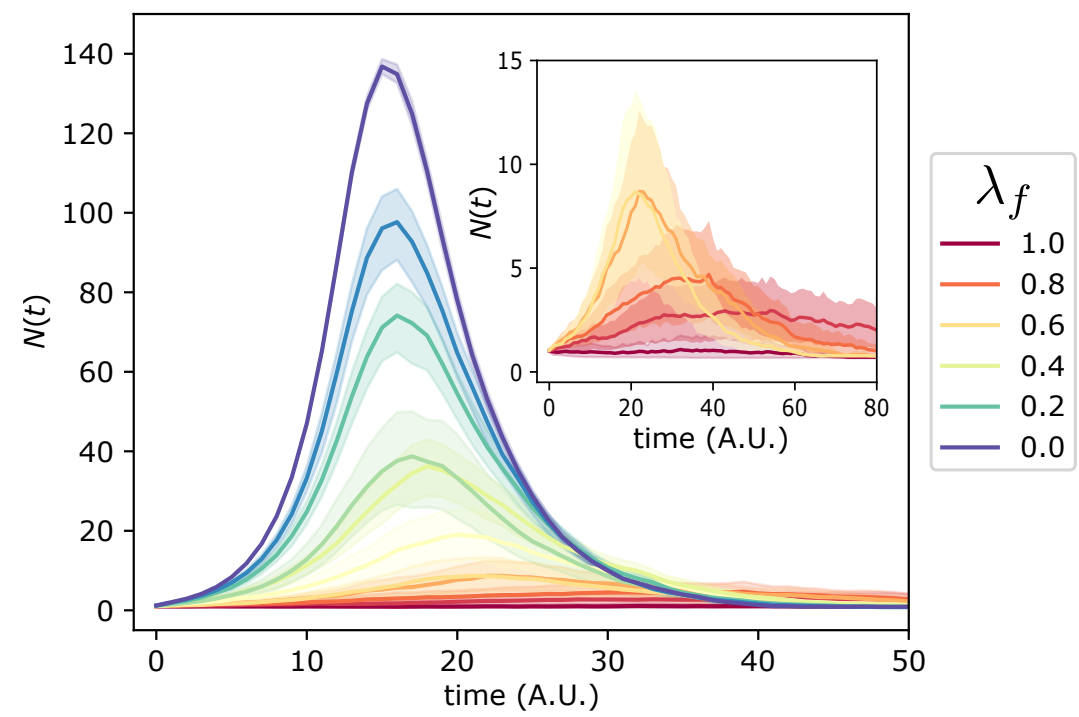

Figure 10: Effects of intrinsic fitness in the boom-and-bust model. For different values of $\lambda_{f}$ we show the temporal dynamics of culture production $(N(t))$. As an inset, we show the dynamics of the higher $\lambda_{f}$ values, i.e. when there is a very large intrinsic value of the products not linked to their novelty. Each solid line represents the average value of 25 independent realizations of the model and the shaded region encompasses one standard deviation of the distribution.

presence of previously unobserved components. (2) The global urn exploration and reinforcement rates reflect changes in the population of creators and their strategies. (3) A coupling across the market population dynamics takes place, i.e. the artifact producer population grows or shrinks proportionally to the average product fitness and the current population size.

In order to challenge the first assumption let's consider the case in which the product fitness is not only determined by the presence of previously unobserved components but also has a random fitness contribution representing its intrinsic value as a product. To this end we will modify Algorithm 2 and introduce a random chance $\left(\lambda_{f}\right)$ of overriding a product fitness with a random uniform value between $\xi \in[-1,1]$ (the same range that a regular fitness value could attain). Figure 10 shows the temporal dynamics of our modified model for different values of $\lambda_{f}$. Essentially, as long as $40 \%$ of the fitness is attributed by innovation, there is a clear boom-and-bust in culture productivity, albeit a 


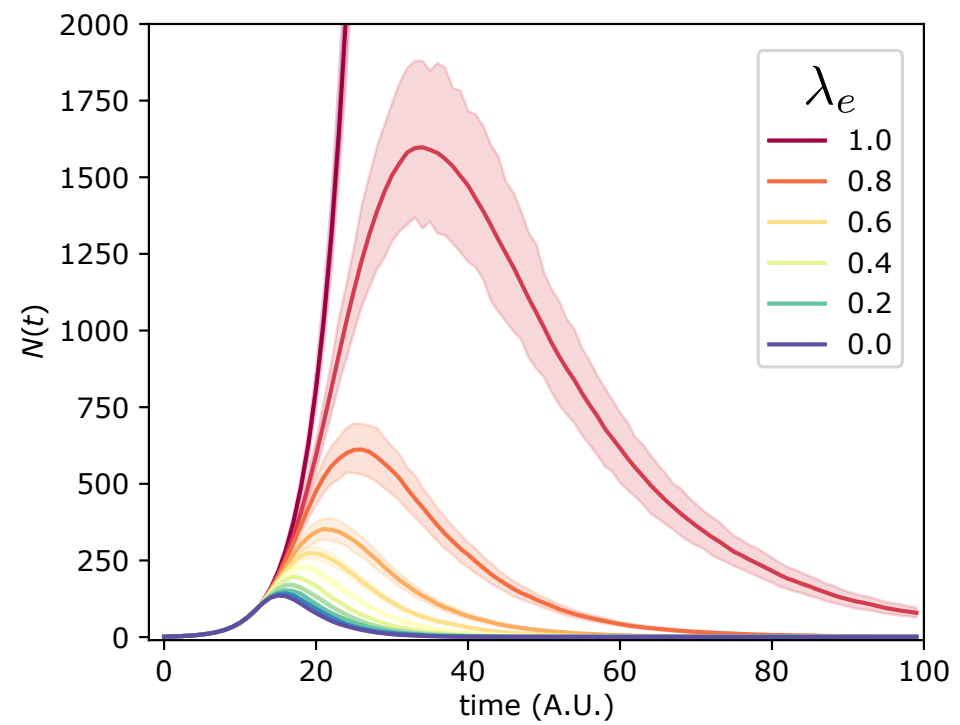

Figure 11: Effects of exploration and reinforcement link to the population in the boom-and-bust model. For different values of $\lambda_{e}$ we show the temporal dynamics of culture production $(N(t))$. Only in the limit case of complete detachment from the actual innovator and imitator population the system keeps growing indefinitely. Each solid line represents the average value of 25 independent realizations of the model and the shaded region encompasses one standard deviation of the distribution.

lower peak $N(t)$ values. When a larger fraction of fitness comes from intrinsic value, there is still a growth and decay which is not steep and with a associated randomness in the time series as large as the peak productivity itself.

For the second assumption let's consider the case in which the urn exploration and reinforcement rates are not exclusively tied to the innovator and imitator population. We will introduce random chance $\left(\lambda_{e}\right)$ of expanding the adjacent possible $(\mathscr{A})$ and the observed components of the urn $(\mathscr{U})$ with random uniform variables whose value scales with the total population $(\xi \in[0, x+y])$. Figure 11 shows the temporal dynamics of our modified model for different values of $\lambda_{e}$. As random exploration becomes more prevalent the peak productivity is delayed and increased in magnitude. Only a the limit of pure random exploration $\left(\lambda_{e}=1\right)$ the system does not collapse and keeps growing indefinitely, meaning that only a very weak linear coupling between the population compart- 


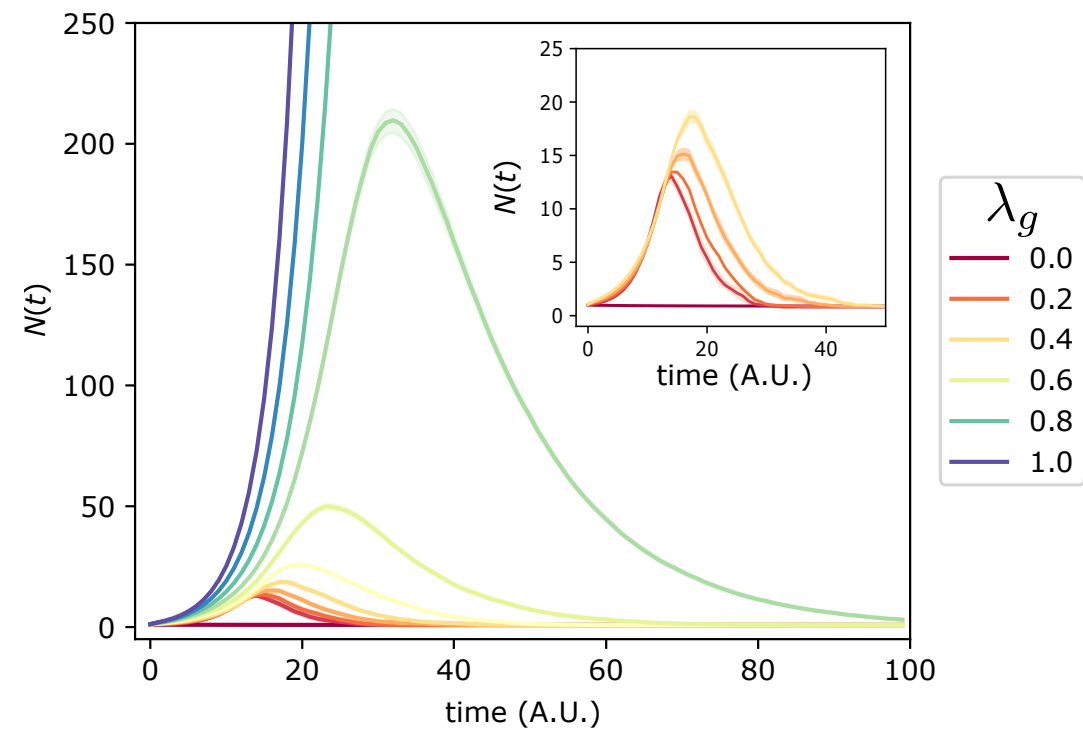

Figure 12: Effects of global population decoupling in the boom-and-bust model.

For different values of $\lambda_{g}$ we show the temporal dynamics of culture production $(N(t))$. As an inset, we show the dynamics of lower $\lambda_{g}$ values, i.e. when imitators are recruited into the system faster than innovators. Each solid line represents the average value of 25 independent realizations of the model and the shaded region encompasses one standard deviation of the distribution.

ments and the urn parameters is necessary in order to obtain a boom-and-bust pattern.

Finally, in order to relax the last premise of the model, we will explore a modification of algorithm 2 in which growth between compartments is not perfectly coupled. Namely, the growth of innovators will be multiplied by a factor $\lambda_{g}$ while the growth of imitators will be scaled by a factor $1-\lambda_{g}$. Figure 12 shows how $N(t)$ varies as $\lambda_{g}$ changes from 0 to 1 . In this case, asymmetry towards imitation reduces peak productivity and makes the collapse happen sooner, while increased innovator growth can make the system never collapse once a certain asymmetry has been reached. 


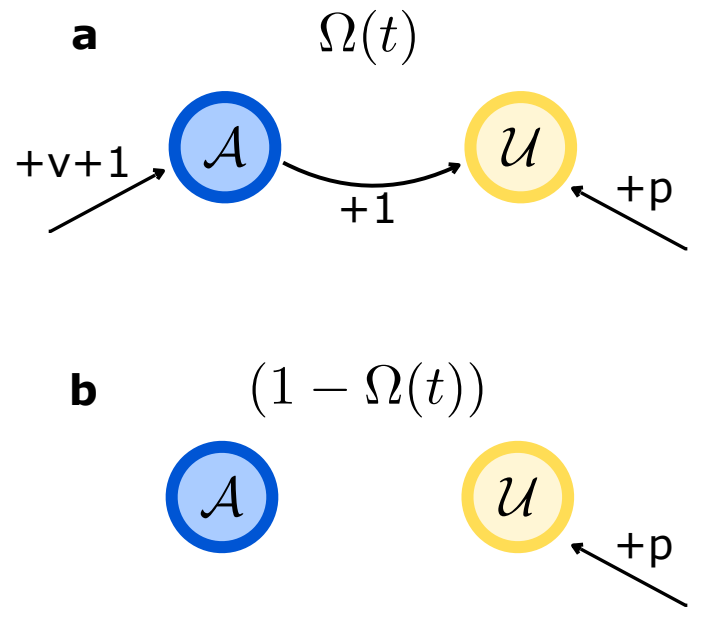

Figure 13: Reactions in the two compartment Polya urn. (a) When the selected element has never been observed prior to the sampling (i.e. it belongs to the adjacent possible), it becomes a regular element, $v+1$ new elements are added as adjacent possible and p copies of the originally sampled element are added to $\mathscr{U}$. (b) When the element selected (chance $(1-\Omega(t))$ ) has been observed before, p copies of it are added to $\mathscr{U}$.

\section{Expansion dynamics of the Adjacent Possible}

When calculating the fitness in our discrete time population dynamics model, and in order to compute the likelihood of creating a novel cultural product, it is useful to know size of the adjacent possible grows with respect to the size of the whole urn, given the parameters $p$ and $v$ (or $y, x$ respectively in the case of populations of imitators and innovators). For this purpose, the complete urn system can be described by the dynamics of two compartments: the adjacent possible (the set of components that have never been sampled but are inside the urn, denoted as $\mathscr{A}$ ) and the set of components that have been sampled at least once (denoted as $\mathscr{U}$ ) [41, 42]. Figure 13 shows a schematic representation of these two compartments with arrows representing sampling processes: when a component from the adjacent possible is chosen, it ceases to be part of the adjacent possible and $p$ copies and $v+1$ new components are added to the urn. The rate of each "reaction" is dependent on the ratio of elements in the adjacent possible versus the total amount of components in the urn 


$$
\Omega(t)=\frac{\mathscr{A}(t)}{\mathscr{A}(t)+\mathscr{U}(t)}
$$

The reactions

$$
\begin{gathered}
\emptyset \rightarrow(p+1) \mathscr{U}+v \mathscr{A} \\
\emptyset \rightarrow(p \mathscr{U})
\end{gathered}
$$

take place with rate $\Omega(t)$ and $1-\Omega(t)$, respectively. With these we can define the time derivatives for each compartment as follows:

$$
\begin{gathered}
\frac{d \mathscr{A}}{d t}=v \Omega(t) \\
\frac{d \mathscr{U}}{d t}=p(1-\Omega(t))+(p+1) \Omega(t)=p+\Omega(t) .
\end{gathered}
$$

Given that there are no size limits to the urn, the total size time derivative is:

$$
\frac{d \mathscr{A}}{d t}+\frac{d \mathscr{U}}{d t}=v \Omega(t)+p+\Omega(t)=p+(v+1) \Omega(t) .
$$

And the time integrals for these compartments follow:

$$
\begin{gathered}
\mathscr{A}(t)=\int_{0}^{t} v \Omega(t) d t=v \sum_{o}^{t} \Omega(t) \\
\left.\mathscr{U}(t)=\int_{0}^{t} p+\Omega(t)\right) d t=p t+\sum_{o}^{t} \Omega(t)
\end{gathered}
$$

For the sake of convenience, hereafter we will represent the definite time integral $\int_{0}^{t} \Omega(t) d t$ as $\bar{\Omega}$. Thus of the time evolution of fraction of elements in the urn that belong to the adjacent possible is:

$$
\Omega(t+1)=\frac{\mathscr{A}(t)}{\mathscr{A}(t)+\mathscr{U}(t)}=\frac{v \bar{\Omega}}{p t+(v+1) \bar{\Omega}}
$$

\subsection{Solution for $\bar{\Omega} \approx k t$}

At this point we can apply an Ansatz to understand the kinds of solutions that this equation accepts. Namely, we will consider different time scaling possibilities for the integral $\bar{\Omega}$. First, if there is a fixed point solution $\left(\Omega^{*}=k\right)$, then its time integral can approximated over large $t$ to:

$$
\bar{\Omega}=\int_{0}^{t} \Omega(t) d t \approx \Omega^{*} t
$$


Returning to the time dependent expression of $\Omega(t)$ we obtain:

$$
\Omega^{*}=\frac{v \Omega^{*} t}{(1+v) \Omega^{*} t+p t},
$$

in which the time dependence can be removed and the expression can be rewritten after some algebra as:

$$
\Omega^{*}\left(p+\Omega^{*}(v+1)-v\right)=0 .
$$

This equation accepts two solutions. If there is no adjacent possible to begin with, the system cannot be expanded, i.e., $\Omega^{*}=0$ (as per definition new elements are added to the adjacent possible only if one element of the adjacent possible is chosen by random sampling). The non-trivial solution to the equation is

$$
\Omega^{*}=\frac{v-p}{v+1},
$$

which depends on both $p$ and $v$.

In Figure 14 we show the numerical value of the time-dependence for different sets of $p$ and $v$ (where $v>p$ ). Additionally, we include the analytical solution for the stable stationary fraction of adjacent possible elements in the urn. When $v>p$, there is a positive stable stationary fraction of adjacent possible in the urn, this means that there is a characteristic product size $C$ for which random samplers of the urn can find at least one novelty half of the time:

$$
\left(1-\Omega^{*}\right)^{C}=1 / 2 .
$$

In other words, there is an optimal value $C^{*}$ for the cultural product length that satisfies the novelty clause imposed in our model. Increasing the size of the cultural product beyond this point incurs in more novelty but is not required to produce an artifact with at least one new element. But what happens if $p>v$ ? For those values the stable stationary fraction is negative, though there is no way to obtain to negative amounts of components or fractions. We will find that the following proposed solutions for $\bar{\Omega}$ temporal scaling give us hints as to the kinds of behaviour that we can expect in the parameter region of $p>v$.

\subsection{Solution for $\bar{\Omega} \approx t / \log (t)$}

Lets consider a solution for the $\Omega$ integral that makes it scale as $k t / \log (t)$. For this family of solutions we have, returning to the temporal variation of the 


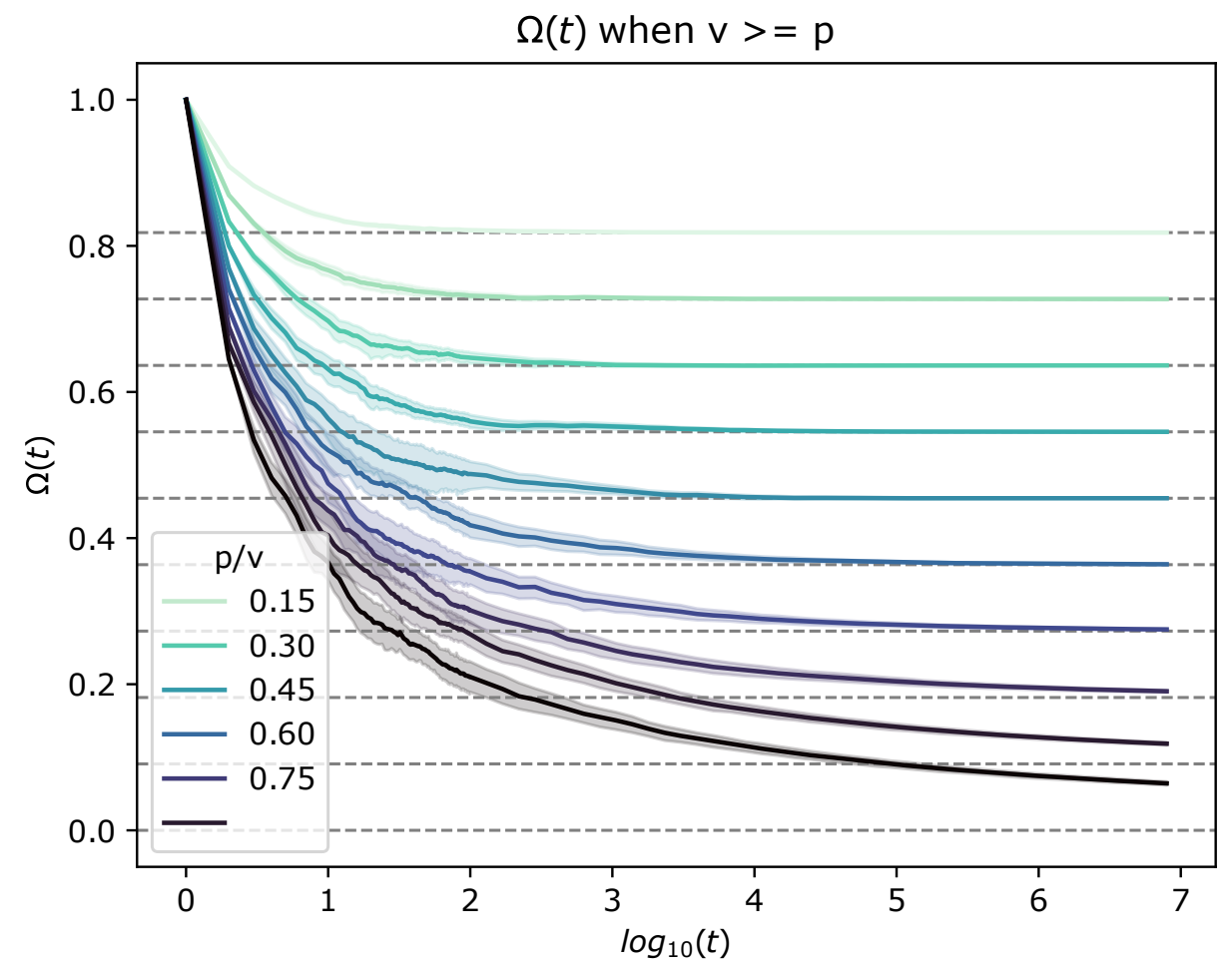

Figure 14: Temporal dynamics of the adjacent possible in a Polia Urn when innovation is stronger than imitation. For different combinations of Urn parameters $(p, v)$ we show the fraction of components in the adjacent possible (i.e. elements that have yet to be used in a cultural product) over time (here $t$ stands for samplings of the urn). As a guide for the eye, we also show as grey dashed lines the corresponding fixed point solutions calculated from $\Omega^{*}=\frac{v-p}{v+1}$. Here the solid line represents the average value of 25 independently simulated time series and the shaded region encompasses one standard deviation of the sample. 
adjacent possible ratio:

$$
\Omega(t)=\frac{v \frac{t}{\log (t)}}{(1+v) \frac{t}{\log (t)}+p t} .
$$

In this case, we can also remove the $t^{0}$ temporal scaling as all terms include this dependence, which leaves us with:

$$
\Omega(t)=\frac{v}{1+v+p \log (t)} .
$$

We find this solution corresponds to the case when $p=v$. Using the solution found previously, the fixed point solution $\Omega^{*}=\frac{v-p}{v+1}$ becomes precisely 0 at $p=v$, which naturally cannot linearly scale with time as per the $\bar{\Omega}$ solution. The numerical integration corresponding to solution is shown in Figure 14 as the last black solid line.

\subsection{Solution for $\bar{\Omega} \approx t^{\alpha}$, where $0<\alpha<1$}

This solution considers a sublineal scaling for $\bar{\Omega}$ using a fractional exponent $0<\alpha<1$. For $\alpha=1$ we converge to the fixed point solution described previously in this section and given that $\Omega(t)$ is bounded to the range $[0,1]$ it cannot scale superlinearly $(\alpha>1)$.

$$
\Omega(t)=\frac{v t^{\alpha}}{(1+v) t^{\alpha}+p t} .
$$

Given that $\alpha$ imposes a sublineal scaling with respect to $p t$, this solution must converge for large $t$ to the simpler equation:

$$
\Omega(t)=\frac{v t^{\alpha}}{p t}=\frac{v}{p} t^{\alpha-1} .
$$

This solution corresponds to the parameter space where $p>v$. As shown in Figure 15 , when $p>v$ no stable fixed point fraction is ever attained, the adjacent possible is dependent on the time of observation (i.e. the amount of samplings that have happened), and keeps becoming smaller with power law time scaling, similarly to the solution presented in this section. In particular we find that the empirical exponent of the adjacent possible over time depends on the Polia urn parameters $(p, v)$ following the expression:

$$
\Omega(t) \sim t^{\frac{v}{p}-1} .
$$



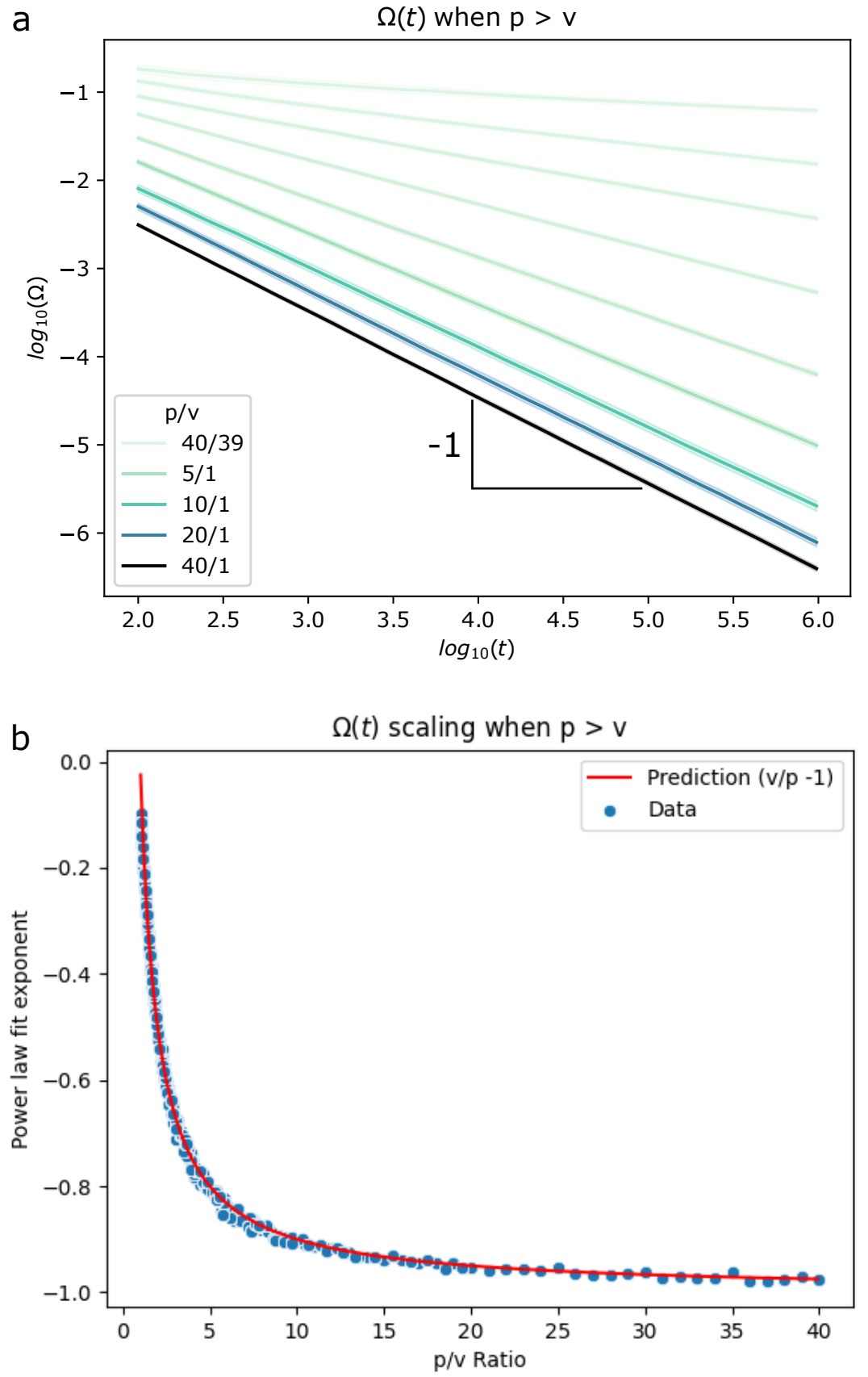

Figure 15: Temporal dynamics of the adjacent possible in a Polia Urn when imitation is stronger than innovation. For different combinations of Urn parameters $(p, v)$ we show haw the fraction of components in the adjacent possible changes over samplings of the urn $t$ (a). Each data point corresponds to the average of 25 independently simulated time series. These change following a power law dependence on time and their empirical exponents are calculated and shown in (b). We also offer a fit for the emp̧ 9 rical exponents depending on the urn parameters, namely $\frac{v}{p}-1$. Each data point here is an independent replicate. 
Figure 15 shows the empirical fit of the power law exponents for simulated urns with different values of $p$ and $v$, represented as a single ratio $p / v$. Since for this part of the parameter space $v / p<1$, the exponent always has negative value.

In this particular solution, the constant shrinking of the adjacent possible could be counteracted by a super-linear scaling of the artifact size over time. This would be mathematically equivalent to extending the search to find novel components, and would be a valid strategy to ensure the production of novelty in this region of parameter space. This would imply an open-ended evolution towards increasingly more complex cultural artifacts, which was observed in the Atari system through the usage of ever larger cartridge sizes (see Section 2).

\subsection{Solution for $\bar{\Omega} \approx k$}

A different type of solution is given by $\bar{\Omega} \approx k$. This type of solution is commonly found in infinite series of the form $S_{\alpha}=\sum_{n=1}^{\infty} \frac{1}{t^{\alpha}}$, where $\alpha>1$, for instance:

$$
S_{\alpha=2}=\sum_{n=1}^{\infty} \frac{1}{t^{2}}=\frac{1}{1}+\frac{1}{4}+\frac{1}{9}+\frac{1}{16}+\cdots=k
$$

while for $\alpha \leq 1$ the infinite series diverges:

$$
S_{\alpha=1}=\sum_{n=1}^{\infty} \frac{1}{t}=\frac{1}{1}+\frac{1}{2}+\frac{1}{3}+\frac{1}{4}+\cdots=\infty .
$$

Returning to the $\Omega(t)$ expression we now have for this family of solutions:

$$
\Omega(t)=\frac{v k}{(1+v) k+p t},
$$

which for large $t$ must converge to $\Omega(t)=\frac{v k}{p} t^{-1}$ and at $\lim _{t \rightarrow \infty} \Omega(t)=$ 0 . This family of solutions actually represent a limit case of $p>>v$ from the previous section, as $p$ becomes much larger than $v$ the temporal scaling in $\Omega(t) \sim t^{\frac{v}{p}-1}$ converges to $\Omega(t) \sim t^{-1}$. 


\subsection{Analytical derivation of $d \Omega / d t$}

For the sake of completeness, we can also derive the analytical expression of $d \Omega / d t$ using the quotient rule:

$$
f^{\prime}(x)=g(x) / h(x)=\frac{g^{\prime}(x) h(x)-g(x) h^{\prime}(x)}{[h(x)]^{2}},
$$

where $f^{\prime}(x)=d \Omega / d t, g(x)=\mathscr{A}(t)$ and $h(x)=\mathscr{A}(t)+\mathscr{U}(t)$. Using the definitions established prior in this section we also know the explicit values for the time derivatives of $\mathscr{A}$ and $\mathscr{U}$ :

$$
\begin{gathered}
d \mathscr{A} / d t=v \Omega(t) \\
d \mathscr{U} / d t=p+\Omega(t) .
\end{gathered}
$$

Then,

$$
\begin{gathered}
d \Omega / d t=\frac{d \mathscr{A} / d t[\mathscr{A}(t)+\mathscr{U}(t)]-\mathscr{A}(t)[d \mathscr{A} / d t+d \mathscr{U} / d t]}{[\mathscr{A}(t)+\mathscr{U}(t)]^{2}} \\
d \Omega / d t=\frac{\Omega(t)[v-(v+1) \Omega(t)-p]}{(v+1) \bar{\Omega}(t)+p t} .
\end{gathered}
$$

\section{Lossless compression of cultural products}

LZW (Lempel-Ziv-Welch) is an universal compression algorithm that builds a word dictionary from the data stream being compressed [43. Consider for example the compression of a text document. At the beginning, the dictionary is initialized with all single-character words (or in the case of video game cartridges, the set of elementary instructions executed by the $6502 \mathrm{CPU})$. LZW reads all characters defined by the text and outputs a new compressed version using only dictionary code words. At each step, a new character is concatenated to the last word read until the new word does not belong to the dictionary. Then, the new code is added to the dictionary, the code is output, and we start again with an empty word. This LZW algorithm is not optimal, but as the text becomes longer, the algorithm becomes asymptotically closer to an optimal compression rate. LZ77 is a related compression algorithm that achieves similar rates as LZW. 


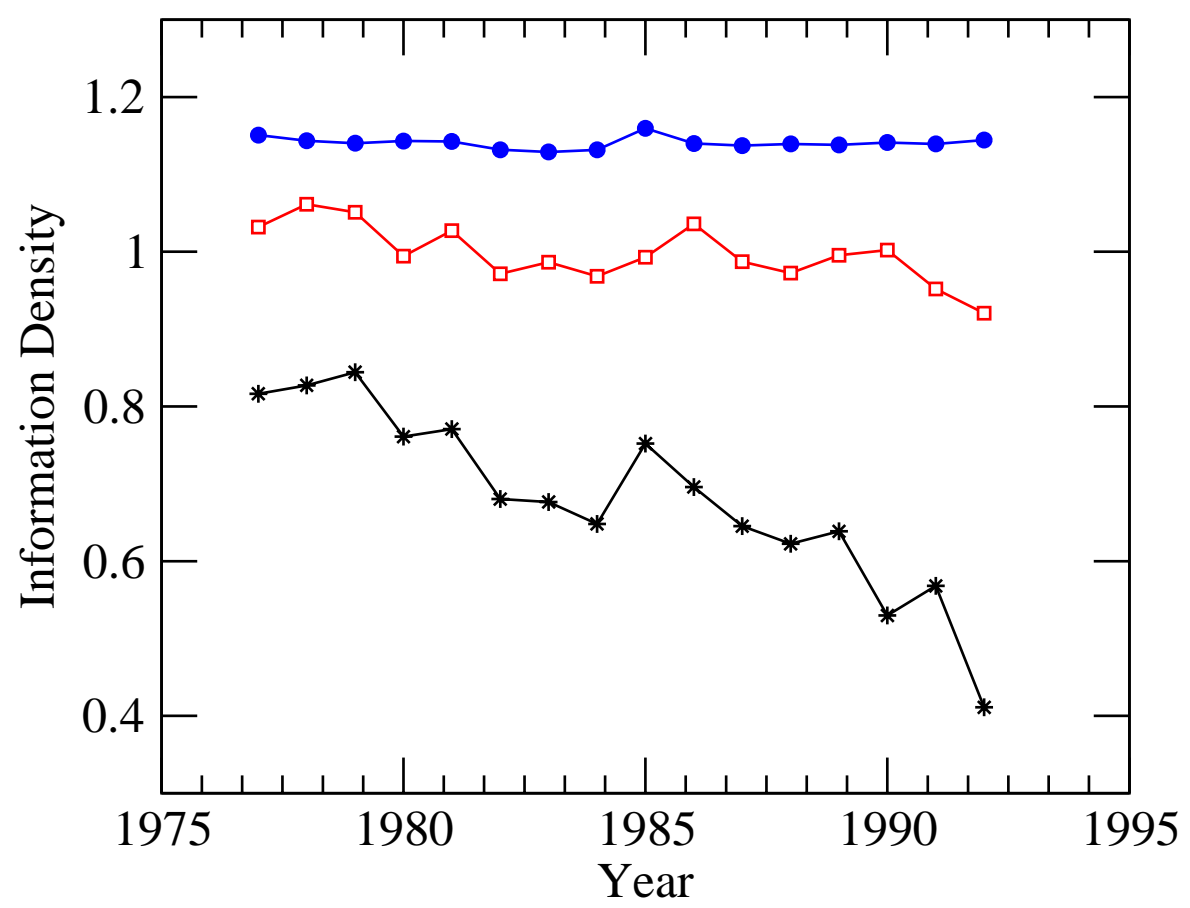

Figure 16: Temporal evolution of compressibility in cultural products computed by LZ77 and normalised by the logarithm of the alphabet size (defined as compressibility in Equation). From bottom to top, we display the evolution of compressibility in three types of sequences, one corresponds to a cultural system while the other two are null-models. Decay of compressibility of a large string concatenating all Atari ROMs released each year (solid black circles). This trend is consistent with a sustained increase of cultural redundancy. On the other hand, this decay of information density is not observed in a large string concatenating all shuffled Atari ROMs released each year (red open circles), which indicates that presence of non-random correlations in cultural systems that cannot be explained with the frequency of byte-codes. Information density of random Fibonacci sequences is constant (solid blue circles). 
Computable information density 44 is proportional to the length of a lossless compressed data string:

$$
\rho=\frac{L(x)}{L}
$$

where $L(x)$ is the total binary code length of the compressed sequence, and $L$ is the length of the original string $x$ (i.e., the number of characters of the string). Information density is not, in general, the same as the compression ratio (or compressibility) of the sequence [45]:

$$
\frac{\rho}{\log (|\alpha|)}
$$

corresponding to the amount of information (bits) per character of the binary representation of the uncompressed string $x$. Here, $\alpha$ is the alphabet of characters used in the string $x$. Information density and compressibility are equivalent for binary sequences (i.e., when $|\alpha|=2$ ).

Compressibility is bounded between 0 and 1 , while information density has no upper bound. The total binary code length $L(x)$ using the LZ77 algorithm [43, 46] can be computed from the number of longest previous factors. These factors are the longest subsequences that we encounter that have already occurred in the past. A factor is defined by a pair $(i, l)$, where $i$ is the starting position of the matching subsequence and $l$ is the subsequence length. A character $c$ that is observed for the first time is represented by the factor $(c, 0)$. The total binary length $L(x)$ of a string $x$ compressed with the LZ77 algorithm is bounded as:

$$
L(x) \leq K \log K+2 K \log \frac{L}{K}+O\left(K \log \log \frac{L}{K}\right)
$$

when $L>|\alpha|$ and all logarithms are taken in base 2 [44. Precise bounds are not known in general [47. It can be shown that LZ77 converges to the optimal compression when $L \rightarrow \infty$ [46]. However, the rate at which optimal compression is achieved is rather slow, in general scaling with the inverse of $\log (L)$. How to extrapolate finite-size measurements of the LZ77 complexity is an open question.

Figure 16 shows the temporal evolution of compressibility computed by LZ77 in a cultural system (Atari ROM cartridges) and two null-models. Deterministic but statistically pseudorandom sequences (like a Fibonacci sequence, cite) approach a compressibility plateau (solid blue circles). Byte-codes occur with 
different probabilities, so even if we compose ROMs by choosing byte-codes independently and at random out of real sequences, its information density will be lower than pseudorandom sequences.

\section{Bipartite network analysis of cultural prod- ucts}

We have also analyzed cultural products as collections of words organized in phrases from very different sources, from natural language technical texts in cryptocurrency to sets of machine instructions stored in ROM cartridges to memes in Reddit. In order for them to be comparable, we have devised a general filtering protocol that allows us to extract from texts their component ideas and relations between them. For natural language texts, we separate the content by phrases, eliminate stop words using the Natural Language Toolkit python library (NLTK version 3.6.1 [48]) and reduce it to semantically related words. This last step of the process was carried out using the WordNet synonym sets database [49, 50, substituting each word by its most commonly used synonym using the Brown corpus and custom Python code.

By collapsing the synonym space we ensure that cultural products are represented by their relevant component ideas instead of considering different synonyms as culturally different elements. This approach has been previously used to more accurately quantify the cognitive extent of science [51] and provide a network evolution model of concepts in innovations [52]. In the case of video games, machine codes are described with sequences of 8-bit (byte) numbers whose specific meaning depends on their location (memory address) in ROM memory. Machine codes are much less ambiguous than natural text to enable efficient processing by the computer processor. These binary codes can be easily understood by humans using the equivalent sequence of assembly code instructions (phrases), which follows a highly simplified grammar. An assembly instruction is encoded with one or more bytes, the first byte indicates the instruction type and the rest of bytes describe the necessary operands (or artificial 'words') to perform the machine operation.

With this collapsed representation of cultural products, we can transform 
a
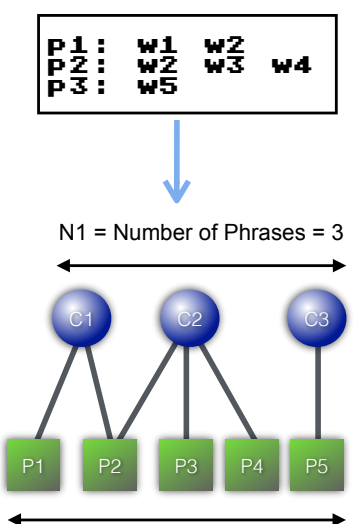

N2 = Vocabulary Size $=5$

\section{C}

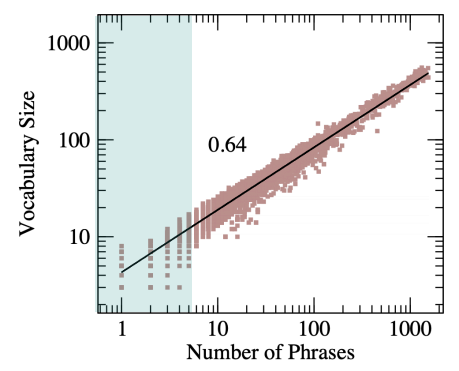

b
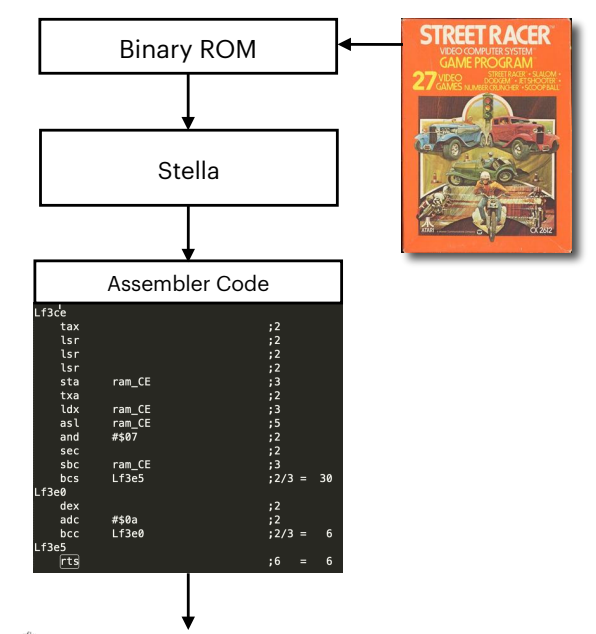

$B_{t}$

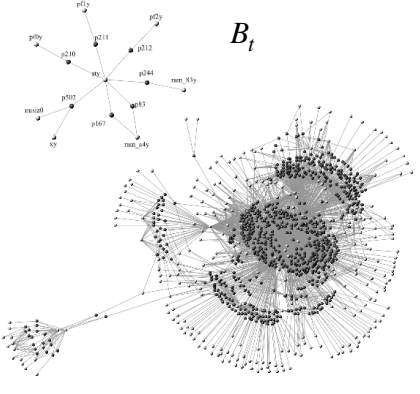

Figure 17: Network analysis of cultural products. (a) We reconstruct a bipartite network $B=\left(V_{1}, V_{2}, E\right)$ (bottom) from the collapsed representation of a cultural product, i.e., a set of phrases $\left(p_{i} \in V_{1}\right)$ composed by words or generally symbols $\left(w_{j} \in V_{2}\right)$ (top). In the bipartite network, there is an edge $\left(p_{i}, w_{j}\right) \in E$ if the word $w_{j}$ appears in the phrase $p_{i}$. (b) Network analysis for the Atari ROM cartridge "Street Racer" (1977). We can use the Stella disassembler (https:// stella-emu.github.io) to translate the binary codes $x$ for this video game to its high-level assembly representation and the list of phrases and words (middle). At the bottom of this panel, we show the reconstructed bipartite network $B$, which displays an heterogeneous structure. (c) Sub-linear scaling of vocabulary size $N_{2} \sim N_{1}^{\alpha}$, where $N_{1}=\left|V_{1}\right|$ is number of phrases and $N_{2}=\left|V_{2}\right|$ is the number of unique words (see text). 
the data from a linear sequence of words into bipartite networks where concepts/words are connected to phrases (see Figure 17a). Using this data structure we can visualize how component ideas relate to each other, and how complex the generative process of these cultural products could be (see Section 11 of the supplementary materials). For example, Figure $17 \mathrm{p}$ shows the network transformation for the video game "Street Racer" developed in 1977 for the Atari 2600 by Larry Kaplan (Atari). Additionally, we analyzed the relation between number of different components and phrase length in the reduced synset data (see Figure 17, ), with a clear scaling exponent close to $\alpha \sim \frac{2}{3}$ for the corresponding Heaps' law in Atari ROMs.

\section{Structural Complexity}

Cultural products evolve from previous objects by means of different mechanisms, like mutation and recombination. Let $X(t)=\left\{x_{0}, x_{1}, x_{2}, \ldots, x_{t}\right\}$ be a "cultural history" or the ordered sequence of cultural or technological products generated from time $t=0$ to time $t$. This evolutionary process is open-ended when the amount of information associated to the evolutionary process is increasing, i.e., the average complexity grows in time:

$$
\left\langle K\left(X_{t}\right)\right\rangle<\left\langle K\left(X_{t}+1\right)\right\rangle .
$$

In general, there will be complexity fluctuations depending on the strength and the trade-offs between evolutionary forces $[53$. We are concerned not only with the possibility of transient drops in complexity, but of cultural collapse, i. e., a sustained decrease of complexity

$$
K\left(x_{t}\right)>K\left(x_{t+1}\right)>\ldots>K\left(x_{\infty}\right)
$$

from time $t$ until the population crashes.

Without any loss of generality, we can consider that cultural systems produce finite binary strings $x_{t}$ at each time step $t$. Strings represent many cultural or technological artefacts, e.g., a text document or the sequence of machine codes stored in a ROM cartridge. We can use the same scheme to encode more complex representations of cultural objects, like bipartite networks or the association 
a

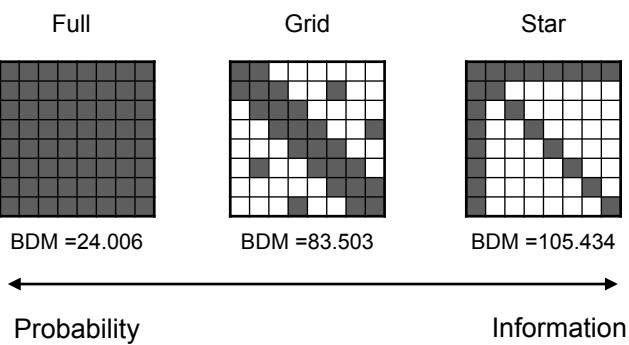

b

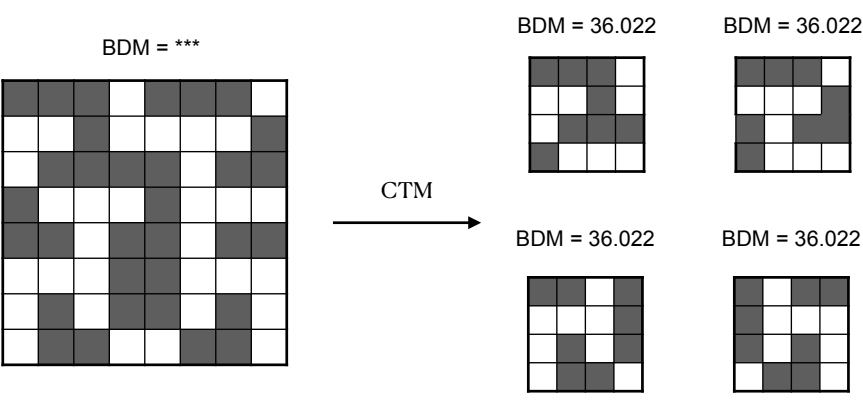

Figure 18: Structural complexity of bipartite networks. (a) From left to right, adjacency matrices for networks of increasing structural complexity. Lowest complexity corresponds to the fully connected network, which can be generated by a very compact program. (b) Due to computational constraints, only the Kolmogorov complexity of small sub-networks can be obtained. For larger networks, we can approximate their structural complexity by decomposing the full network (left) in the smaller sub-networks (right).

between sentences and unique symbols (see Section 10). Given a network of $N$ nodes, we can encode its adjacency matrix with a $N^{2}$-length bit string.

The algorithmic complexity of a binary string $x$ is given by its KolmogorovChaitin complexity $K(x)$, that is, the length of the shortest program generating that string [54, 55, 56]. Algorithmic complexity provides a more robust test for cultural randomness than Shannon entropy. Lossless compression algorithms account for statistical, but not algorithmic regularities [57]. That is, algorithmic complexity can recognize low-complexity strings that look random from a statistical perspective (high entropy). For example, the string 01010101...01 is clearly periodic but displays maximal entropy when looked at the bit level (the frequency of Os and 1s is the same). We can still detect this strong regular- 
ity (and thus minimal entropy) when sub-sequences of 2 bits are the target of analysis. Indeed, a very short program can generate the string by repeatedly concatenating the ' 01 ' motif until the desired length is achieved. In general, lossless compression represents a lower-bound for object complexity because Shannon entropy is not invariant to the level of description [58, i.e., they lack an invariance theorem.

Algorithmic complexity is uncomputable [56]. Fortunately, the Coding Theorem Method provides good approximations of algorithmic complexity $K(x)$ for short binary strings $x[59,60$. This is based on the relationship between Kolmogorov complexity and the algorithmic probability (also known as Solomonoff probability) 61. The algorithmic probability $m(x)$ of a string $x$ is the probability that a random program $p$ generates the string $x$ :

$$
m(x)=\sum_{p: \mathscr{U}(p)=x} \frac{1}{2^{|p|}}
$$

where $\mathscr{U}(p)$ is the string generated by an universal Turing machine $\mathscr{U}[62,63$. when running the program $p$, and $|p|$ is the number of program instructions. The algorithmic Coding Theorem relates the quantities $m(x)$ and $K(x)$ :

$$
|-\log m(x)-K(x)|<c
$$

where $c$ is a constant value that is independent of the string [64]. This implies the more frequent a string is, the lower its Kolmogorov complexity:

$$
K(x)=-\log m(x)+O(1) .
$$

This theorem suggests how to estimate the Kolmogorov complexity of a string from the frequency of random programs producing it. However, an important obstacle is the number of Turing machines is astronomically large. By a combination of theoretical and experimental results, 65. generated all Turing machines with up to four states. This approach was later extended to Turing machines with 5 states and 2 symbols, and thus providing an accurate estimation for the complexity $K(x)$ of all binary strings of length $|x| \leq 16$ bits $[66$.

Furthermore, we can use the CTM to approximate the complexity of large strings. The Block Decomposition Method (BDM) divides any large string into 
smaller sub-strings (for which the CTM provides a complexity approximation), and then puts together these local complexity values to obtain the complexity of the full string [57. This approach can also be applied with bipartite networks, where the BDM decomposes the target adjacency matrix into smaller (and possibly overlapping) $4 \times 4$ subgraphs (see Figure 18b). The structural complexity $\operatorname{BDM}(G)$ of the bipartite network $G$ is computed as follows:

$$
B D M(G)=\sum_{G_{i} \subseteq G} K\left(G_{i}\right)+\log \left(N_{i}\right)
$$

where $G_{i} \subseteq G$ represent the different (isomorphic) types of subgraphs, $N_{i}$ is the number of occurrences of the subgraph $G_{i}$ found in $G$, and $K\left(G_{i}\right)$ is the two-dimensional Kolmogorov complexity as computed using the CTM 66, 67. We compute the normalized structural complexity $B D M(G)$ using the Python package PyBDM [68] version 0.1.0, and normalize it using the following factor:

$$
N B D M(G)=\frac{B D M(G)}{L \log _{2}(|\alpha|)},
$$

where $L$ stands for the bipartite adjacency matrix size in the network representation $G$. That is, the vocabulary size multiplied by the number of phrases of a given cultural product. For a binary representation of a link in a bipartite network (no weights) the alphabet is $\alpha=\{0,1\}$, i.e. the number of different symbols present $(|\alpha|)$ is 2 .

\subsection{Coarse-grained analysis of collated posts in the red- dit data sets}

In the main text analysis of normalized structural complexity $(N B D M)$, we find that both the control and boom-and-bust data set of social media posts display fairly constant average complexities. This observation in the temporal pattern is validated in Figure 21, where we show a statistical test for the NBDM distribution across the observational periods. Although the structural complexity approximated by PyBDM clearly recapitulates some temporal patterns in larger cultural products, equations 1 and 2 in the previous section remind us that there is an inescapable constant in the approximation of algorithmic complexity that is independent of the string or string size. The impact of such 

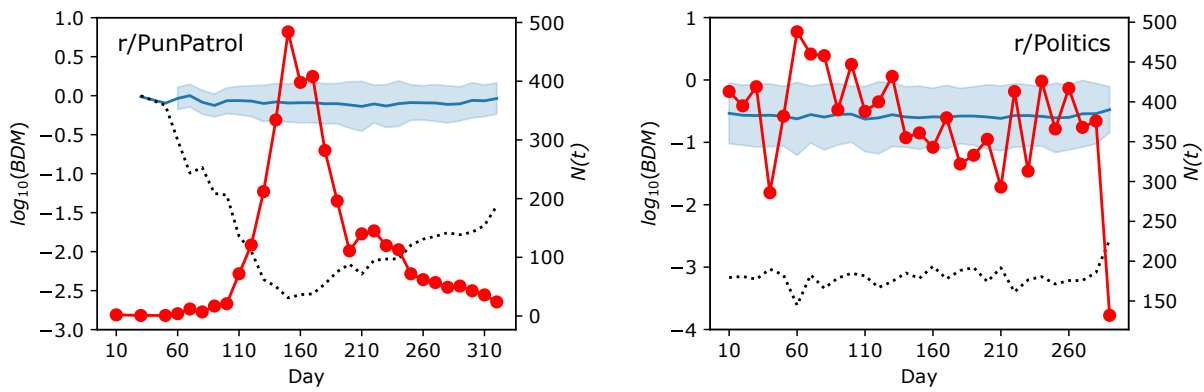

Figure 19: Temporal pattern of structural complexity in the coarse-grained reddit datasets. For r/PunPatrol and r/Politics we aggregated the posts for any given observational period (10 day bins) and computed the singular NBDM of that period. Blue solid line represents the average complexity as shown in the main text, red solid line follows the cultural diversity for any given period and dashed black line represents the $N B D M$ of the aggregated posts for that temporal slice.

constant will be larger the lower the intrinsic complexity of the structure we are trying to quantify. In order to test this perspective, we aggregated all the cultural products pertaining to a given observational period (slices of 10 days for both the $\mathbf{r}$ /PunPatrol and $\mathbf{r} /$ Politics data sets) and computed the sizenormalized structural complexity of the aggregate cultural product. Figure 19 shows how in this context, $\mathbf{r} /$ Politics does not display any trend in time while the $\mathbf{r}$ /PunPatrol temporal sequence seems to be coupled with the boom-andbust pattern in a non-trivial way. Although this coarse-graining precludes us from determining the significance of the observed pattern, it suggests that the reduction in complexity is indeed present in this example although difficult to observe in the smaller scales of individual social media posts. 

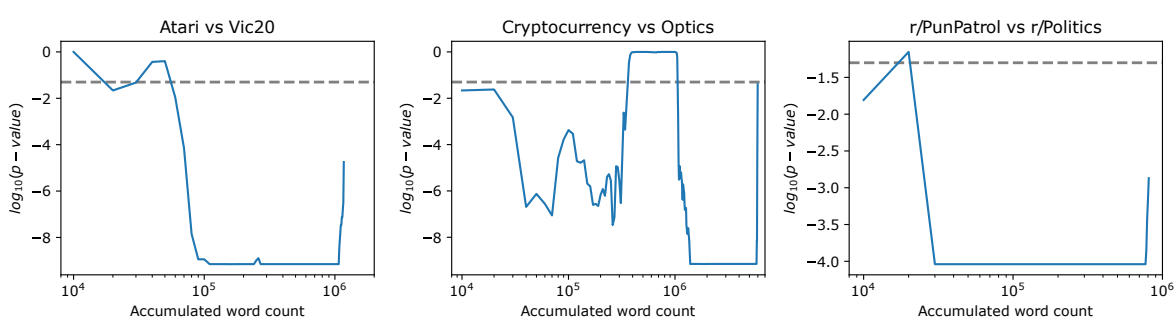

Figure 20: Statistical significance of mean exponent differences. For each pair of datasets we evaluate whether the observed differences exponent values in a given accumulated word count are statistically significant, i.e. whether they come from the same distribution. Mann-Whitney U test results are shown as the $\log _{10}$ (p-value), additionally, as a guide for the eye, the commonly used $p=0.05$ threshold for significance is shown as a dashed grey line.

\section{Statistical analysis of the hallmark metrics of imitation}

\subsection{Zipf's law exponents}

In this section we discuss the methodology used to assess statistical significance of the temporal patterns of frequency rank exponent, compressiblity and complexity variation. In all cases a non-parametric test was carried out (MannWhitney U [69], scipy stats python library [70], version 1.6.2). Without assuming normality of the data, we examined the hypothesis that one of the samples had a lower mean value than the other. In the case of the word frequency rank exponent distributions, we tested the hypothesis that the controls (Vic-20, scientific publications in the field of Optics and $\mathbf{r}$ /Politics) had lower mean values than our boom-and-bust datasets. Figure 20 shows that the hypothesis holds true in all three experiments for large sections of the time series, extending even further than the periods highlighted in the main text. However, the statistical significance is particularly high during the boom-and-bust cycle, reaching $p=10^{-8.81}$ in the Atari vs. Vic-20 comparison, $p=10^{-9.15}$ in the cryptocurrency vs. scientific publications and $p=10^{-4.04}$ in the $\mathbf{r} / \mathbf{P u n P a t r o l}$ analysis. 


\subsection{Compressibility}

Figure ?? shows the results of non-parametric test (Mann-Whitney U 69], scipy stats python library [70, version 1.6.2) confirming that statistical significance of the decay of compressibility in all three datasets when compared to the negative controls.

\subsection{Artifact complexity}

In the case of cultural product complexity, we tested the hypotesis that Atari, cryptocurrency and $\mathbf{r}$ /PunPatrol harbored less complex cultural products as time progressed during the boom-and-bust cycle, i.e. that the observed decrease average $\log _{10}(N B D M)$ had statistical significance. In the same vein as before, we used a non-parametric test (Mann-Whitney U 69], scipy stats python library [70, version 1.6.2) comparing the period $\log _{10}(N B D M)$ distribution between temporal segments (years or 10-day periods). As Figure 21 shows, during the booming years of productivity, both Atari games and cryptocurrency white papers were structurally less complex than the earlier products. Additionally, in the case of cryptocurrency, there is a loss of significance and upwards trend in the later periods after the bust, suggesting a correction in the dynamics of imitation in the system towards more innovation. In the case of $\mathbf{r} / \mathbf{P u n P a t r o l}$ posts, no significant trends were observed.

\section{References}

[1] Anne Kandler and Enrico R Crema. Analysing cultural frequency data: neutral theory and beyond. In Handbook of evolutionary research in archaeology, pages 83-108. Springer, 2019.

[2] Henry Lowood. Videogames in computer space: The complex history of pong. IEEE Annals of the History of Computing, 31(3):5-19, 2009.

[3] Nick Montfort and Ian Bogost. Racing the beam: The Atari video computer system. Mit Press, 2009.

[4] Lance A Leventhal. 6502 assembly language programming. McGraw-Hill, Inc., 1986. 

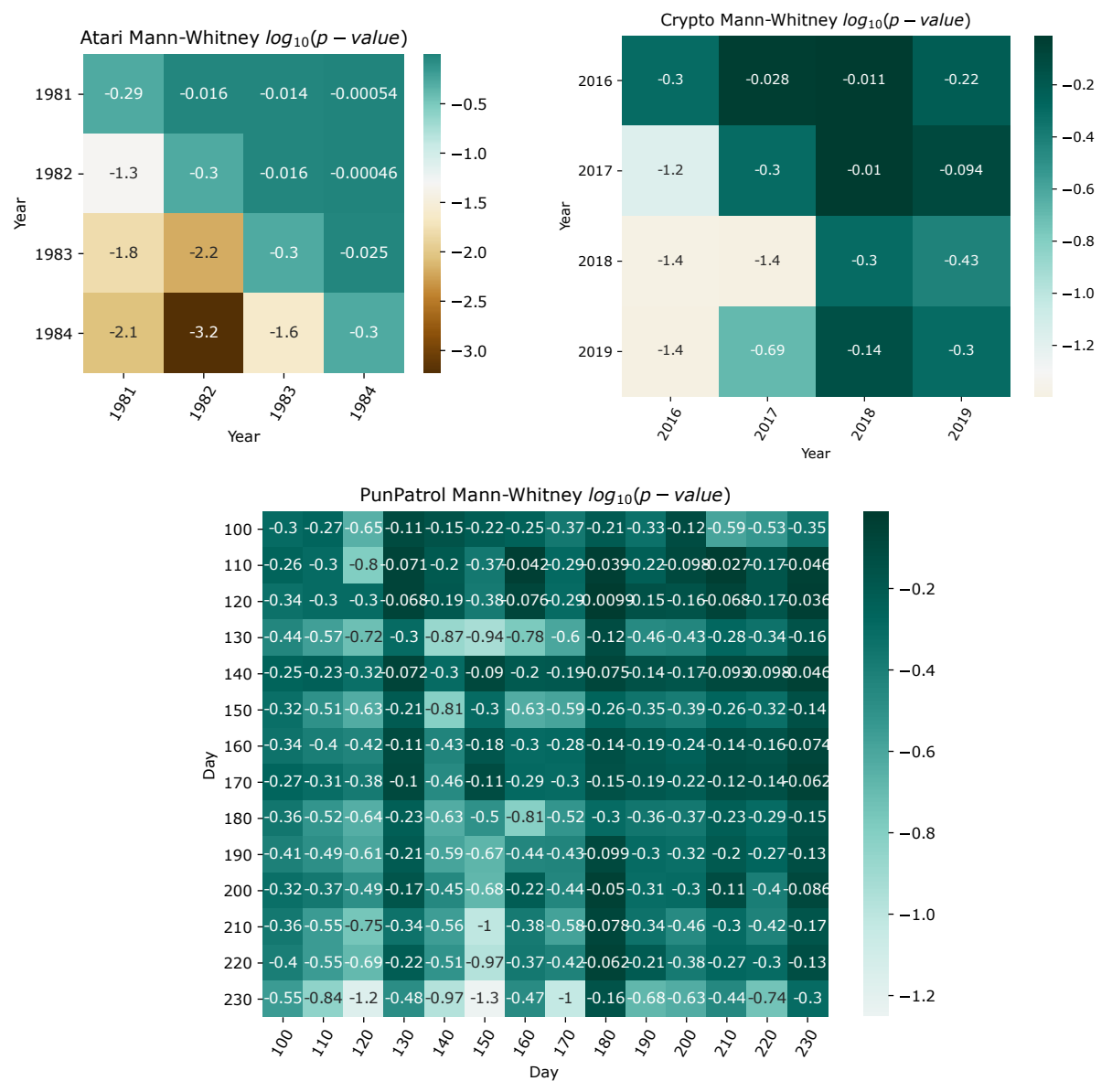

Figure 21: Statistical significance of $\log _{10}(N B D M)$ evolution across domains. For each domain we evaluate whether there are significant differences in the $\log _{10}(N B D M)$ distribution between years. Mann-Whitney results are shown as the $\log _{10}(\mathrm{p}$-value), testing whether the segment on the y-coordinate had lower mean value than the period on the $\mathrm{x}$-coordinate.

[5] Michael E Porter. Competitive strategy: Techniques for analyzing industries and competitors, 1980 .

[6] Mark Paich and John D Sterman. Boom, bust, and failures to learn in experimental markets. Management science, 39(12):1439-1458, 1993.

[7] Bruce Smith. VIC 20 Machine Code. Shiva Publishing Limited, 1984. URL https://archive.org/details/VIC-20_Machine_Code 
[8] Satoshi Nakamoto. Bitcoin: A peer-to-peer electronic cash system. Technical report, Manubot, 2008.

[9] Harald Vranken. Sustainability of bitcoin and blockchains. Current opinion in environmental sustainability, 28:1-9, 2017.

[10] Ferdinando M Ametrano. Hayek money: The cryptocurrency price stability solution. Available at SSRN 2425270, 2016.

[11] Max J Krause and Thabet Tolaymat. Quantification of energy and carbon costs for mining cryptocurrencies. Nature Sustainability, 1(11):711-718, 2018.

[12] Jonathan Koomey. Estimating bitcoin electricity use: A beginner's guide. May, Coin Center Report, https://www. coincenter. org/app/uploads/2020/05/estimating-bitcoinelectricity-use. pdf, 2019.

[13] Sinan Küfeoğlu and Mahmut Özkuran. Bitcoin mining: A global review of energy and power demand. Energy Research 63 Social Science, 58:101273, 2019.

[14] Michel Rauchs, A Blandin, and A Dek. Cambridge bitcoin electricity consumption index (cbeci), 2020.

[15] Camilo Mora, Randi L Rollins, Katie Taladay, Michael B Kantar, Mason K Chock, Mio Shimada, and Erik C Franklin. Bitcoin emissions alone could push global warming above 2 c. Nature Climate Change, 8(11):931-933, 2018.

[16] Christian Stoll, Lena Klaaßen, and Ulrich Gallersdörfer. The carbon footprint of bitcoin. Joule, 3(7):1647-1661, 2019.

[17] Abeer ElBahrawy, Laura Alessandretti, Anne Kandler, Romualdo PastorSatorras, and Andrea Baronchelli. Evolutionary dynamics of the cryptocurrency market. Royal Society open science, 4(11):170623, 2017.

[18] Elie Bouri, Georges Azzi, and Anne Haubo Dyhrberg. On the returnvolatility relationship in the bitcoin market around the price crash of 2013. Economics, 11(1), 2017. 
[19] Sergei Tikhomirov, Ekaterina Voskresenskaya, Ivan Ivanitskiy, Ramil Takhaviev, Evgeny Marchenko, and Yaroslav Alexandrov. Smartcheck: Static analysis of ethereum smart contracts. In Proceedings of the 1st International Workshop on Emerging Trends in Software Engineering for Blockchain, pages 9-16, 2018.

[20] Usman W Chohan. Bitconnect and cryptocurrency accountability. Available at SSRN 3131512, 2018.

[21] Ruozhou Liu, Shanfeng Wan, Zili Zhang, and Xuejun Zhao. Is the introduction of futures responsible for the crash of bitcoin? Finance Research Letters, 34:101259, 2020 .

[22] Mar 2020. URL https://whitepaperdatabase.com/.

[23] Mar 2020. URL https://www.allcryptowhitepapers.com/

[24] Mar 2020. URL https://whitepaper.io/

[25] Mar 2020. URL https://icosbull.com/

[26] Apr 2020. URL https://textract.readthedocs.io/en/stable/.

[27] Mar 2020. URL https://icoholder.com/.

[28] Mar 2020. URL https://icorating.com/.

[29] Apr 2020. URL https://www. coingecko.com/.

[30] Apr 2020. URL https://data.world/datasets/cryptocurrency.

[31] Mar 2020. URL https://pubmed.ncbi.nlm.nih.gov/

[32] Maria Glenski, Corey Pennycuff, and Tim Weninger. Consumers and curators: Browsing and voting patterns on reddit. IEEE Transactions on Computational Social Systems, 4(4):196-206, 2017.

[33] Adrienne Massanari. \# gamergate and the fappening: How reddit's algorithm, governance, and culture support toxic technocultures. New media Es society, 19(3):329-346, 2017. 
[34] Annika Richterich. 'karma, precious karma!' karmawhoring on reddit and the front page's econometrisation. Journal of Peer Production, 4(1), 2014.

[35] Carlo Michele Valensise, Matteo Cinelli, Alessandro Galeazzi, and Walter Quattrociocchi. Drifts and shifts: Characterizing the evolution of users interests on reddit. arXiv preprint arXiv:1912.09210, 2019.

[36] Richard Dawkins. The selfish gene. Oxford university press, 2016.

[37] May 2020. URL https://praw.readthedocs.io/en/latest/.

[38] Francesca Tria, Vittorio Loreto, Vito Domenico Pietro Servedio, and Steven H Strogatz. The dynamics of correlated novelties. Scientific reports, 4(1):1-8, 2014.

[39] Elena Miu, Ned Gulley, Kevin N Laland, and Luke Rendell. Flexible learning, rather than inveterate innovation or copying, drives cumulative knowledge gain. Science advances, 6(23):eaaz0286, 2020.

[40] Mario Coccia and Joshua Watts. A theory of the evolution of technology: Technological parasitism and the implications for innovation magement. Journal of Engineering and Technology Management, 55:101552, 2020.

[41] W Brian Arthur, Yu M Ermoliev, and Yu M Kaniovski. Path-dependent processes and the emergence of macro-structure. European journal of operational research, 30(3):294-303, 1987.

[42] W Brian Arthur et al. Increasing returns and path dependence in the economy. University of michigan Press, 1994.

[43] Jacob Ziv and Abraham Lempel. A universal algorithm for sequential data compression. IEEE Transactions on information theory, 23(3):337-343, 1977.

[44] Stefano Martiniani, Paul M Chaikin, and Dov Levine. Quantifying hidden order out of equilibrium. Physical Review X, 9(1):011031, 2019.

[45] Jacob Ziv and Abraham Lempel. Compression of individual sequences via variable-rate coding. IEEE transactions on Information Theory, 24(5): $530-536,1978$. 
[46] Paul C Shields. Performance of lz algorithms on individual sequences. IEEE Transactions on Information Theory, 45(4):1283-1288, 1999.

[47] Paul C Shields. Universal redundancy rates do not exist. IEEE transactions on information theory, 39(2):520-524, 1993.

[48] Steven Bird, Ewan Klein, and Edward Loper. Natural language processing with Python: analyzing text with the natural language toolkit. " O'Reilly Media, Inc.", 2009.

[49] George A Miller. Wordnet: a lexical database for english. Communications of the ACM, 38(11):39-41, 1995.

[50] Maria Ruiz-Casado, Enrique Alfonseca, and Pablo Castells. Automatic assignment of wikipedia encyclopedic entries to wordnet synsets. In International Atlantic Web Intelligence Conference, pages 380-386. Springer, 2005.

[51] Staša Milojević. Quantifying the cognitive extent of science. Journal of Informetrics, 9(4):962-973, 2015.

[52] Iacopo Iacopini, Staša Milojević, and Vito Latora. Network dynamics of innovation processes. Physical review letters, 120(4):048301, 2018.

[53] Alexander Neef, Amparo Latorre, Juli Pereto, Francisco J Silva, Miguel Pignatelli, and Andres Moya. Genome economization in the endosymbiont of the wood roach cryptocercus punctulatus due to drastic loss of amino acid synthesis capabilities. Genome biology and evolution, 3:1437-1448, 2011.

[54] Andrei N Kolmogorov. On tables of random numbers. Theoretical Computer Science, 207(2):387-395, 1998.

[55] Gregory J Chaitin. On the length of programs for computing finite binary sequences. Journal of the ACM (JACM), 13(4):547-569, 1966.

[56] Ming Li, Paul Vitányi, et al. An introduction to Kolmogorov complexity and its applications, volume 3. Springer, 2008. 
[57] Hector Zenil, Santiago Hernández-Orozco, Narsis A Kiani, Fernando SolerToscano, Antonio Rueda-Toicen, and Jesper Tegnér. A decomposition method for global evaluation of shannon entropy and local estimations of algorithmic complexity. Entropy, 20(8):605, 2018.

[58] Hector Zenil, Narsis A Kiani, and Jesper Tegnér. Low-algorithmiccomplexity entropy-deceiving graphs. Physical Review E, 96(1):012308, 2017.

[59] Nicolas Gauvrit, Hector Zenil, Jean-Paul Delahaye, and Fernando SolerToscano. Algorithmic complexity for short binary strings applied to psychology: a primer. Behavior research methods, 46(3):732-744, 2014.

[60] Fernando Soler-Toscano, Hector Zenil, Jean-Paul Delahaye, and Nicolas Gauvrit. Correspondence and independence of numerical evaluations of algorithmic information measures. Computability, 2(2):125-140, 2013.

[61] Ray J Solomonoff. A preliminary report on a general theory of inductive inference. Citeseer, 1960.

[62] Alan Mathison Turing. On computable numbers, with an application to the entscheidungsproblem. Proceedings of the London mathematical society, 2 (1):230-265, 1937.

[63] Sanjeev Arora and Boaz Barak. Computational complexity: a modern approach. Cambridge University Press, 2009.

[64] Thomas M Cover and Joy A Thomas. Information theory and statistics. Elements of Information Theory, 1(1):279-335, 1991.

[65] Jean-Paul Delahaye and Hector Zenil. Numerical evaluation of algorithmic complexity for short strings: A glance into the innermost structure of randomness. Applied Mathematics and Computation, 219(1):63-77, 2012.

[66] Fernando Soler-Toscano, Hector Zenil, Jean-Paul Delahaye, and Nicolas Gauvrit. Calculating kolmogorov complexity from the output frequency distributions of small turing machines. PloS one, 9(5):e96223, 2014. 
[67] Hector Zenil, Fernando Soler-Toscano, Jean-Paul Delahaye, and Nicolas Gauvrit. Two-dimensional kolmogorov complexity and an empirical validation of the coding theorem method by compressibility. PeerJ Computer Science, 1:e23, 2015.

[68] Sept 2020. URL https://pybdm-docs.readthedocs .io/en/latest/.

[69] Henry B Mann and Donald R Whitney. On a test of whether one of two random variables is stochastically larger than the other. The annals of mathematical statistics, pages 50-60, 1947.

[70] Pauli Virtanen, Ralf Gommers, Travis E Oliphant, Matt Haberland, Tyler Reddy, David Cournapeau, Evgeni Burovski, Pearu Peterson, Warren Weckesser, Jonathan Bright, et al. Scipy 1.0: fundamental algorithms for scientific computing in python. Nature methods, 17(3):261-272, 2020. 